NEW ZEALAND JOURNAL OF MATHEMATICS

Volume 52 (2021), 605-642

https://doi.org/10.53733/141

\title{
ENERGY-MINIMAL PRINCIPLES IN GEOMETRIC FUNCTION THEORY
}

\author{
Tadeusz Iwaniec, Gaven Martin, and Jani Onninen \\ (Received 21 July 2021) \\ In memory of Sir Vaughan Frederick Randal Jones \\ Kua hinga te tōtara i te wao nui a Tāne
}

\begin{abstract}
We survey a number of recent developments in geometric analysis as they pertain to the calculus of variations and extremal problems in geometric function theory following the NZMRI lectures given by the first author at those workshops in Napier in 1998 and 2005.
\end{abstract}

\section{Introduction}

This article is a reflection of the authors' research program to advance variational techniques in Geometric Function Theory with many and varied applications in mind. On one hand to things like nonlinear materials science, the calculus of variations, nonlinear analysis and PDEs, and on the other to Teichmüller theory and Riemann surfaces. While making a few new observations, the material in this article is largely expository in nature and presents a number of recent advances from the NZMRI lectures given by the first author at those workshops in Napier in 1998 (Geometric Analysis) and 2005 (Interactions between Geometry and Algebra). Indeed it was during that second conference that we (with Kari Astala - also in attendence) began [3] which set up the main initial problems in the area, identified the connections between minimisers of mean distortion and harmonic mappings and the Nitsche phenomenon, $[\mathbf{3 5}, \mathbf{2}]$. These ultimately led to the resolution of the Nitsche Conjecture from 1962, [20]. Generalisations were discussed in [22].

The modern theory seeks an in-depth analysis of deformations which minimise naturally occurring energy functionals in geometric analysis and solve the associated Euler-Lagrange (and other related) equations. Crucially, we confine ourselves not just to examples, but to use these examples to gain insight and new points of view to uncover novel phenomena - such as the Nitsche phenomenon, which unexpectedly identified conformal invariants as obstructions to the existence of 'nice' minimisers for even the Dirichlet energy, see (1.2) below. Thus the main objects of our discussion are mappings

$$
h: \mathbb{X} \stackrel{\text { onto }}{\longrightarrow} \mathbb{Y}
$$

between given spaces of the same topological type; the map $h$ is typically, an orientation preserving homeomorphism and referred to as an elastic deformation in materials science. Although $\mathbb{X}$ and $\mathbb{Y}$ could be general Riemannian $n$-manifolds (with or without boundaries), we shall largely focus on the case they are Euclidean

2020 Mathematics Subject Classification 30C62 31A05 49J10.

The research of the TI \& JO supported by the U.S.A. National Science Fund, GJM was supported by the N.Z. Marsden Fund. 
domains $\mathbb{X}, \mathbb{Y} \subset \mathbb{R}^{n}$. Our standing assumption is that $h$ belongs to some Sobolev space $\mathscr{W}_{\text {loc }}^{1, p}\left(\mathbb{X}, \mathbb{R}^{n}\right)$; where normally $1<p<\infty$. The case $p=1$ will often be treated as marginal due to the lack of reflexibility of $\mathscr{W}^{1,1}\left(\mathbb{X}, \mathbb{R}^{n}\right)$; it turns out that reflexibility is a fundamental prerequisite for establishing the existence of energy-minimal mappings (usually called hyperelastic deformations) via existence approaches based in the direct method of the calculus of variations. Typically a studied problem will require a priori bounds in a natural Sobolev space. For instance in the classical case of determining the hyperelastic deformation of the energy functional

$$
h \mapsto \int_{\mathbb{X}}|D h(x)|^{2} d \sigma_{\mathbb{X}}(x)
$$

subject to any constraints, one must seek minimisers in $\mathscr{W}^{1,2}(\mathbb{X}, \mathbb{Y})$. Here

$$
|D h(x)|^{2}=\sum_{i, j}\left|\frac{\partial h^{j}}{\partial x_{k}}\right|^{2}, \quad x=\left(x_{1}, \ldots, x_{N}\right) \in \mathbb{X}, \quad h(x)=\left(h_{1}(x), \ldots, h_{N}(x)\right) \in \mathbb{Y}
$$

is the Hilbert-Schmidt norm.

Sobolev mappings between Riemannian manifolds can be defined in several ways, that are not necessary equivalent. We may, and do, assume that $\mathbb{Y}$ is a subset (not necessarily a subdomain) of $\mathbb{R}^{N}$ for some sufficiently large dimension $N$. This follows from the celebrated embedding theorem of J. Nash [34]. This being so, we say that $h: \mathbb{X} \rightarrow \mathbb{Y} \subset \mathbb{R}^{N}$ belongs to $\mathscr{W}^{1, p}(\mathbb{X}, \mathbb{Y})$ if each of its $N$ coordinate functions lies in the linear space $\mathscr{W}^{1, p}(\mathbb{X}, \mathbb{R})$ and $h(x) \in \mathbb{Y}$ for every $x \in \mathbb{X}$; here there is a standard way of defining Sobolev scalar functions on a manifold. However, caution must be exercised because the topology of the target space $\mathbb{Y}$ (later referred to as the deformed configuration) may prevent smooth mappings from being dense in $\mathscr{W}^{1, p}(\mathbb{X}, \mathbb{Y}),[\mathbf{1 8}]$.

The following problem, still open, gives a glimpse of the difficulties arising already at the very basic stages concerning Sobolev homeomorphisms.

Problem 1.1. Let $\mathbb{Y} \subset \mathbb{R}^{n}$ be a bounded topological ball (so homeomorphic to the unit ball $\mathbb{B}$ ). For what $p$ (any ?) does there exist a homeomorphism $h: \mathbb{B} \stackrel{\text { onto }}{\longrightarrow} \mathbb{Y}$ of Sobolev class $\mathscr{W}^{1, p}(\mathbb{B}, \mathbb{Y})$ ?

\section{Sobolev Homeomorphisms as Elastic Deformations}

Elastic deformations of material bodies have fascinated famous scientists for centuries as one can see in the writings of, for instance, G. Galileo, H. Hook, L. Euler, J-L. Lagrange, T. Young, A. Cauchy, G. Green, and G.R. Kirchoff. Nowadays, the study of mathematical models for nonlinear elasticity is a very active science. Here we will not cover all the major developments of the modern theory, but will discuss the principles with a determination to rework, using other recent mathematical advances, some of the problematic presuppositions. At the heart of these presuppositions is the Principle of Noninterpenetration of Matter, [5]. This roughly asserts that an energy minimiser should always be a homeomorphism (and perhaps even a diffeomorphism depending on the problem - but certainly not discontinous, see $[6])$. Here we will see that one must really adopt the more general class of monotone $(n=2)$ Sobolev mappings as legitimate hyperelastic deformations of elastic 
bodies. This leads to the "Weak Principle of Noninterpenetration of Matter" and even in this apparently weaker context new topological and geometrical arguments are essential.

2.1. Hyperelasticity. For the mathematical models of nonlinear elasticity, we study homeomorphisms $h: \mathbb{X} \stackrel{\text { onto }}{\longrightarrow} \mathbb{Y}$ of smallest stored energy,

$$
\mathscr{E}[h]=\int_{\mathbb{X}} \mathbf{E}(x, h, D h) d x, \quad \mathbf{E}: \mathbb{X} \times \mathbb{Y} \times \mathbb{R}^{n \times n} \rightarrow \mathbb{R}
$$

where the so-called stored energy function $\mathbf{E}$ characterizes the various mechanical and elastic properties of the materials occupying the domains $\mathbb{X}$ and $\mathbb{Y}$. We have also written $d x$ as a shorthand for whatever measure $d \sigma_{\mathbb{X}}(x)$ is given as data on $\mathbb{X}$. The p-harmonic energy,

$$
\mathscr{E}_{p}[h]=\int_{\mathbb{X}}|D h(x)|^{p} d x
$$

including the Dirichlet integral $(p=2)$, is the ideal example to illustrate the scheme for the direct method in the Calculus of Variations, and we will do so later after we introduce a few more natural stored energy functionals.

When $p \geq 1$ the integrand in (2.2) is a convex function of the differential $D h$. We will soon see why this is important. The case $p=n$ will hereafter be referred to as the Conformal Energy. This is due to the fact that $\mathscr{E}_{n}[h]=\int_{\mathbb{X}}|D h(x)|^{n} d x$ is invariant under a conformal change of the $x$-variable.

Let us also introduce two other natural functionals. First the so-called $b i$ conformal energy defined for homeomorphisms $h: \mathbb{X} \stackrel{\text { onto }}{\longrightarrow} \mathbb{Y}$ of Sobolev class $\mathscr{W}^{1, n}(\mathbb{X}, \mathbb{Y})$ whose inverse $f \stackrel{\text { def }}{=} h^{-1}: \mathbb{Y} \stackrel{\text { onto }}{\longrightarrow} \mathbb{X}$ also lies in the Sobolev space $\mathscr{W}^{1, n}(\mathbb{Y}, \mathbb{X})$

$$
\mathscr{E}_{n}[h, f]=\int_{\mathbb{X}}|D h(x)|^{n} d x+\int_{\mathbb{Y}}|D f(y)|^{n} d y
$$

This can be formulated, equivalently via the change of variable $y=h(x)$ in the second integral, by means of one polyconvex energy functional for $h$ on $\mathbb{X}$ :

$$
\mathscr{T}[h]=\int_{\mathbb{X}}\left(|D h(x)|^{n}+\left|(D h(x))^{-1}\right|^{n} J(x, h)\right) d x
$$

Here $J(x, h)$ is the Jacobian determinant of $D h$ and polyconvexity, discussed further below, refers to the fact that the integrand is again a convex function of the minors of the differential $D h$. We don't assert that it is obvious that $\left|(D h(x))^{-1}\right|^{n} J(x, h)$ or $|D h(x)|^{n} / J(x, h)$ are such convex functions. From the latter we consider the $q$-conformal energy functional

$$
\mathscr{K}_{q}[h]=\int_{\mathbb{X}}\left(\frac{|D h(x)|^{n}}{J(x, h)}\right)^{q} d x .
$$

Here the integrand is actually a distortion function usually denoted

$$
\mathbb{K}(x, h)=\frac{|D h(x)|^{n}}{J(x, h)}
$$

and is an infinitesimal measure of a the anisotropic nature of the deformation. To see this note that for suitably regular deformations $h$ we can order the eigenvalues of $D h^{t} D h$ as $0<\lambda_{1}^{2} \leq \lambda_{2}^{2}<\cdots<\lambda_{n}^{2}<\infty$. Then 


$$
n^{n / 2}\left(\frac{\lambda_{1}}{\lambda_{n}}\right)^{n} \leq \mathbb{K}(x, h)^{2}=\frac{\left(\lambda_{1}^{2}+\lambda_{2}^{2}+\cdots+\lambda_{n}^{2}\right)^{n / 2}}{\lambda_{1} \cdot \lambda_{2} \cdot \cdots \cdot \lambda_{n}} \leq n^{n / 2}\left(\frac{\lambda_{n}}{\lambda_{1}}\right)^{n}
$$

Thus $\mathbb{K}$ is controlled by the linear distortion,

$$
K=K(x, h)=\frac{\lambda_{n}}{\lambda_{1}}=\limsup _{r \rightarrow 0} \frac{\max _{|\zeta|=r}|h(x+\zeta)-h(x)|}{\min _{|\zeta|=r}|h(x+\zeta)-h(x)|}
$$

In two dimensions actually $\mathbb{K}(x, h)=\frac{1}{2}(K+1 / K)$. A $\mathscr{W}^{1, n}(\mathbb{X}, \mathbb{Y})$ homeomorphism between Euclidean domains with $\mathbb{K} \in L^{\infty}(\mathbb{X})$ (equivalently $K \in L^{\infty}(\mathbb{X})$ ) is called quasiconformal. The functional $\mathscr{K}_{q}[h]$ is sometimes referred to as the $q$-mean distortion.

To understand the existence problem for hyperelastic deformations, that is minimisers, we must first accept the weak limits of energy-minimizing sequences of homeomorphisms as legitimate deformations. Thus we allow for weak interpenetration of matter; roughly speaking, squeezing of portion of the material to a point can occur, but not folding or tearing. This potentially changes the nature of a minimisation problem to the extent that the minimal energy among such weaker deformations might be strictly smaller than the infimum energy among homeomorphisms. Indeed this can happen. However when it does not, one might subsequently attempt to explain why this squeezing doesn't happen. A classical example of this topological regularity is the Rado-Kneser-Choquet theorem for harmonic mappings from $1926 \&$ 1945, $[\mathbf{9}, \mathbf{2 6}, \mathbf{3 6}]$. This simply states that a homeomorphic harmonic mapping is a diffeomorphism. The non-vanishing of the Jacobian determinant is real thing that needs to be proved. This result has been extended in various ways, see $[\mathbf{2 9}]$ and the references therein.

Different kinds of variational problems occur naturally in geometric function theory. The first and most important example of course is the Riemann mapping theorem which we now consider in the above context.

2.2. Conformal mappings are frictionless-minimisers of Dirichlet energy. Frictionless refers to problems where we do not prescribe what a minimiser is to be on the boundary of $\mathbb{X}$. We are merely given the domains $\mathbb{X}$ and $\mathbb{Y}$ as "configurations".

Theorem 2.1. Let $h: \mathbb{X} \stackrel{\text { onto }}{\longrightarrow} \mathbb{Y}$ be a conformal map between bounded domains $\mathbb{X}, \mathbb{Y} \subset \mathbb{R}^{2} \simeq \mathbb{C}$. Then every orientation preserving homeomorphism $f: \mathbb{X} \stackrel{\text { onto }}{\longrightarrow} \mathbb{Y}$ of Sobolev class $\mathscr{W}^{1,2}(\mathbb{X}, \mathbb{C})$ has Dirichlet energy at least that of $h$. Equality occurs if and only if $f$ is conformal as well.

Proof. Using complex variables $z=x_{1}+i x_{2} \in \mathbb{X}, \frac{\partial}{\partial z}=\frac{1}{2}\left(\frac{\partial}{\partial x}-i \frac{\partial}{\partial y}\right)$ and so forth, the statement reads as follows

$$
\begin{aligned}
\mathscr{E}_{2}[h] & =2 \int_{\mathbb{X}}\left|h_{z}\right|^{2}+\left|h_{\bar{z}}\right|^{2}=2 \int_{\mathbb{X}}\left|h_{z}\right|^{2}-\left|h_{\bar{z}}\right|^{2}=2 \int_{\mathbb{X}} J(z, h) d z \\
& =2|\mathbb{Y}|=2 \int_{\mathbb{X}} J(z, f) d z=2 \int_{\mathbb{X}}\left|f_{z}\right|^{2}-\left|f_{\bar{z}}\right|^{2} \leqslant \mathscr{E}_{2}[f]
\end{aligned}
$$

Equality occurs if and only if $f_{\bar{z}}=0$, which from Weyl's lemma implies that $f$ : $\mathbb{X} \stackrel{\text { onto }}{\longrightarrow} \mathbb{Y}$ is conformal. 
Here we have used the fact that both Jacobians $J(z, h)=\left|h_{z}\right|^{2}-\left|h_{\bar{z}}\right|^{2}$ and $J(z, f)=$ $\left|f_{z}\right|^{2}-\left|f_{\bar{z}}\right|^{2}$ have the same integral over $\mathbb{X}$, namely the area of $\mathbb{Y}$. This property is given to more general nonlinear differential expressions called Free Lagrangians we discuss later.

Remark 2.2. As remarked above, conformal mappings are solutions to the first order differential equations $f_{\bar{z}}=0$, originally introduced by D'Alembert and traditionally referred to as the Cauchy-Riemann equations. By contrast, the variational equations for the stored energy at (2.1) are second order PDEs (in this case the Laplacian) which is usually subject to prescribed boundary values $h_{0}: \partial \mathbb{X} \rightarrow \partial \mathbb{Y}$. Typically in the Riemann mapping problem we are only given the initial domain $\mathbb{X}=\mathbb{D}$ and the target domain $\mathbb{Y}$ (that is the shape of the deformed configuration) without specifying the boundary values of $h$ - determining how the boundary should be deformed. Of course $\mathbb{X}$ may also be any simply connected domain.

With boundary conditions the minimisation problem would be ill posed. That is one cannot prescribe the boundary values of a conformal mappings. The minimisation problem would yield a solution within harmonic deformations, but whose real and imaginary parts need not be harmonic conjugates of one another.

In fact, this is among the simplest and most natural examples of a general frictionless problem in the calculus of variations. Frictionless problems concern energyminimal deformations $h_{\circ}: \mathbb{X} \stackrel{\text { onto }}{\longrightarrow} \mathbb{Y}$ (usually homeomorphisms) with no prescribed boundary map $h_{\circ}: \partial \mathbb{X} \stackrel{\text { onto }}{\longrightarrow} \partial \mathbb{Y}$; in other words, tangential slipping along $\partial \mathbb{X}$ is allowed. In nonlinear elasticity this is physically realised when deforming confined incompressible material. The use of the direct method for these sorts of problems has brought us to the concept of Free Lagrangians. However first, let us briefly outline the evolving concept of Null Lagrangians, extensively discussed and developed in the celebrated paper [7]. That paper includes the references for the many significant earlier contributions to this idea.

\section{Direct Method for $p$-Harmonic Energy.}

As noted above, a representative example for convex functionals is the $p$-harmonic energy of mappings $h: \mathbb{X} \rightarrow \mathbb{R}^{m}$ with prescribed boundary values.

$$
\mathscr{E}_{p}[h]=\int_{\mathbb{X}}|D h(x)|^{p} \mathrm{~d} x, \quad h \in h_{\circ}+\mathscr{W}_{0}^{1, p}\left(\mathbb{X}, \mathbb{R}^{m}\right), 1<p<\infty .
$$

Here, the given mapping $h_{\circ} \in \mathscr{W}^{1, p}\left(\mathbb{X}, \mathbb{R}^{m}\right)$ takes the role of boundary data in the weak formulation of the Dirichlet problem. The function space $\mathscr{W}_{0}^{1, p}$ refers to those functions vanishing on the boundary (in the Sobolev sense - the closure of $\mathscr{C}_{0}^{\infty}(\mathbb{X})$ in the appropriate norm).

We note the following aspects of the setup here.

- We are naturally using the separable reflexive Banach space

$$
\mathfrak{B}=\mathscr{W}^{1, p}\left(\mathbb{X}, \mathbb{R}^{m}\right)
$$

- The functional $\mathscr{E}_{p}$, subject to minimization, is defined on a subset

$$
\mathfrak{B}_{\circ} \stackrel{\text { def }}{=} h_{\circ}+\mathscr{W}_{0}^{1, p}\left(\mathbb{X}, \mathbb{R}^{m}\right)
$$

which is closed in the weak topology of $\mathfrak{B}$. 
- Coercivity Condition. We have a condition which controls the $\mathfrak{B}$-norm of $f \in \mathfrak{B}_{\circ}$ by means of its energy $\mathscr{E}_{p}[f]$. In the above example, we have a routine estimate

$\|f\|_{\mathfrak{B}}^{p} \stackrel{\text { def }}{=} \int_{\mathbb{X}}|D f|^{p}+|f|^{p} \sim\left\|h_{\circ}\right\|_{\mathfrak{B}}^{p}+\mathscr{E}_{p}[f]$, for every $f \in \mathfrak{B}$

- An energy-minimizing sequence has a limit. That $h_{\kappa} \in \mathfrak{B}_{\circ}, \kappa=1,2, \ldots$, means that

$$
\inf \left\{\mathscr{E}_{p}[h] ; h \in \mathfrak{B}_{\circ}\right\}=\lim \mathscr{E}_{p}\left[h_{\kappa}\right] .
$$

Since $\mathfrak{B}$ is reflexive, we may extract from $\left\{h_{\kappa}\right\}$ a subsequence, still denoted by $\left\{h_{\kappa}\right\}$, converging weakly to a mapping $h_{\infty} \in \mathfrak{B}_{\circ}$.

- Lower semicontinuity. Now, everything hinges on establishing the inequality

$$
\mathscr{E}[f] \leqslant \liminf _{i \rightarrow \infty} \mathscr{E}\left[f_{i}\right] \text {, whenever } f_{i} \rightarrow f \text {, weakly in } \mathfrak{B}
$$

Here we are only interested in (3.3) for energy minimising sequences, but as a general property the sequence $\left\{f_{i}\right\}$ need not be energy-minimizing. Customary terminology refers to those energy functionals satisfying (3.3) as being sequentially weakly lower semicontinuous. For simplicity we omit the words "sequentially weakly". The route to establishing lower semicontinuity usually goes through a subgradient estimate.

- Subgradient Estimate. For an integrand $\mathbf{E}(x, y, \xi)$ that is convex with respect to $\xi \in \mathbb{R}^{m \times n}$ we have a subgradient estimate:

$$
\mathbf{E}(x, y, \xi)-\mathbf{E}\left(x, y, \xi_{\circ}\right) \geqslant\left\langle\nabla_{\xi} \mathbf{E}\left(x, y, \xi_{\circ}\right) \mid \xi-\xi_{\circ}\right\rangle
$$

Here the symbol $\langle\mid\rangle$ stands for the inner product of $m \times n$-matrices. In our $p$-harmonic example this inequality, upon integration, reads as

$$
\int_{\mathbb{X}}|D f(x)|^{p} d x-\int_{\mathbb{X}}\left|D f_{\circ}(x)\right|^{p} d x \geqslant p \int_{\mathbb{X}}\left\langle\left|D f_{\circ}\right|^{p-2} D f_{\circ} \mid D f-D f_{\circ}\right\rangle
$$

Now we take $f=h_{\kappa}$ and $f_{\circ}=h_{\infty}$. Then $D h_{\kappa}-D h_{\infty}$ converges weakly to zero in the space $\mathscr{L}^{p}\left(\mathbb{X}, \mathbb{R}^{m \times n}\right)$ and $\left|D f_{\circ}\right|^{p-2} D f_{\circ}$ lies in the dual space $\mathscr{L}^{q}\left(\mathbb{X}, \mathbb{R}^{m \times n}\right)$, where $\frac{1}{p}+\frac{1}{q}=1$. Hence

as required.

$$
\int_{\mathbb{X}}\left|D h_{\kappa}(x)\right|^{p} d x \rightarrow \int_{\mathbb{X}}\left|D h_{\infty}(x)\right|^{p} d x
$$

Remark 3.1. This natural illustration of the direct method actually has wideranging enhancements in which the role of weak convergence $D h_{\kappa} \rightarrow D h_{\infty}$ is taken by Null Lagrangians. We discuss these next.

\section{Null Lagrangians and Polyconvex Functionals}

4.1. Null Lagrangians. Consider a Sobolev mapping $f=\left(f^{1}, \ldots, f^{m}\right): \mathbb{X} \rightarrow \mathbb{R}^{m}$ of an open region $\mathbb{X} \subset \mathbb{R}^{n}$ into $\mathbb{R}^{m}$. The term null Lagrangian pertains to a nonlinear differential $n$-form $\mathbf{N}(x, f, D f) d x$, whose integral mean over any open subregion $\Omega \subset \mathbb{X}$ depends only on the boundary values of $f: \partial \Omega \rightarrow \mathbb{R}^{m}$. While there are technicalities here such as defining a Sobolev function on $\partial \Omega$, typically these things are not at issue when $f$ is at least continuous. 
This condition is reminiscent of that for exact differential forms, by virtue of Stokes' formula. It may very well be right to call such expressions nonlinear exact forms. Distinctive examples are furnished by the subdeterminants of the $m \times n$ matrix of the linear tangent map $D f: \mathbb{R}^{n} \stackrel{\text { into }}{\longrightarrow} \mathbb{R}^{m}$ called the deformation gradient,

$D f(x) \stackrel{\text { def }}{=}\left[\begin{array}{cccc}\frac{\partial f^{1}}{\partial x_{1}} & \frac{\partial f^{1}}{\partial x_{2}} & \ldots & \frac{\partial f^{1}}{\partial x_{n}} \\ \frac{\partial f^{2}}{\partial x_{1}} & \frac{\partial f^{2}}{\partial x_{2}} & \ldots & \frac{\partial f^{2}}{\partial x_{n}} \\ \vdots & \vdots & & \vdots \\ \frac{\partial f^{m}}{\partial x_{1}} & \frac{\partial f^{m}}{\partial x_{2}} & \ldots & \frac{\partial f^{m}}{\partial x_{n}}\end{array}\right]=\left[\frac{\partial f^{i}}{\partial x_{j}}\right] \in \mathbb{R}^{m \times n}, i=1, \ldots, m, j=1, \ldots, n$

To every pair $(I, J)$ of ordered $\ell$-tuples $I: 1 \leqslant i_{1}<i_{2}<\ldots<i_{\ell} \leqslant m$ and $J: 1 \leqslant j_{1}<j_{2}<\ldots<j_{\ell} \leqslant n$, with $1 \leq \ell \leqslant \min \{m, n\}$, there corresponds an $\ell \times \ell$ -minor of $D f(x)$, denoted by

$$
\frac{\partial f^{I}}{\partial x_{J}}=\frac{\partial\left(f^{i_{1}}, \ldots, f^{i_{\ell}}\right)}{\partial\left(x_{j_{1}}, \ldots, x_{j_{\ell}}\right)}
$$

These minors, are the coefficients of the wedge product:

$$
d f^{i_{1}} \wedge \ldots \wedge d f^{i_{\ell}}=\sum_{1 \leqslant j_{1}<\ldots<j_{\ell} \leqslant n} \frac{\partial\left(f^{i_{1}}, \ldots, f^{i_{\ell}}\right)}{\partial\left(x_{j_{1}}, \ldots, x_{j_{\ell}}\right)} d x_{j_{1}} \wedge \ldots \wedge d x_{j_{\ell}}=\sum_{J} \frac{\partial f^{I}}{\partial x_{J}} d x_{J}
$$

Stokes' formula tells us that for $f, g \in \mathscr{W}^{1, \ell}\left(\Omega, \mathbb{R}^{m}\right)$ we have

$$
\int_{\Omega} \frac{\partial f^{I}}{\partial x_{J}} \mathrm{~d} x=\int_{\Omega} \frac{\partial g^{I}}{\partial x_{J}} \mathrm{~d} x, \quad \text { provided } f-g \in \mathscr{W}_{\circ}^{1, \ell}\left(\Omega, \mathbb{R}^{m}\right)
$$

This leads to the affine combinations (with constant coefficients) of the Jacobian subdeterminants as examples of null Lagrangians

$$
\mathbf{N}(x, f, D f)=\sum_{\ell=0}^{\min \{m, n\}} \sum_{\substack{1 \leqslant i_{1}<\ldots<i_{\ell} \leqslant m \\ 1 \leqslant j_{1}<\ldots<j_{\ell} \leqslant n}} \lambda_{i_{1} \ldots i_{\ell}}^{j_{1} \ldots j_{\ell}} \frac{\partial\left(f^{i_{1}}, \ldots, f^{i_{\ell}}\right)}{\partial\left(x_{j_{1}}, \ldots, x_{j_{\ell}}\right)} \stackrel{\text { def }}{=} \mathbf{N}(D f)
$$

where we adhere to the convention that the term with $\ell=0$ is a constant function. In fact we have the following interesting characterisation.

Theorem 4.1. Formula (4.3) represents all null Lagrangians of the form

$$
\mathbf{N}(x, f, D f) d x=\mathbf{N}(D f) d x
$$

This result goes back to $[\mathbf{2 7}, \mathbf{1 1}, \mathbf{1 5}, \mathbf{3 7}$. Should it be required to appeal to a first order null Lagrangians of the general form $E(x, f, D f) d x$, we refer to the work by de Franchis [10].

The utility of null Lagrangians is best illustrated for polyconvex functionals discussed next. 
4.2. Polyconvexity. In his mathematical models for nonlinear elasticity [4] J. Ball made the crucial observation that if the convexity of the stored energy integrand $\mathbf{E}(x, h, D h)$, with respect to the deformation gradient $D h(x) \in \mathbb{R}^{m \times n}$, must be ruled out, it could be replaced by a weaker requirement; namely, expressing the integrand as a convex function of all of the subdeterminants of $D h$,

$$
\mathbf{E}(x, h, D h)=\mathbf{E}_{\mathbf{H}}(x, h, \text { subdeterminants of } D h)
$$

The number of all possible $\ell \times \ell$-subdeterminants with $0 \leqslant \ell \leqslant \min \{m, n\}$ is equal to

$$
\sum_{\ell=0}^{\min \{m, n\}}\left(\begin{array}{c}
m \\
\ell
\end{array}\right)\left(\begin{array}{l}
n \\
\ell
\end{array}\right)=\left(\begin{array}{c}
m+n \\
n
\end{array}\right)
$$

Thus we are assuming that for every pair $(x, y) \in \mathbb{X} \times \mathbb{Y}$ the function

$$
\mathbf{E}_{\text {w }}(x, y, \cdots) ; \mathbb{R}^{\left(\begin{array}{c}
m+n \\
n
\end{array}\right)} \rightarrow \mathbb{R},
$$

is convex. The idea of minimizing polyconvex energy functionals is based on a quite far reaching extension of the direct method of the calculus of variations that we outlined earlier. It has turned out that so far this is the only practical idea that offers substantially more than that of just minimising convex energies, for instance the $p$-harmonic example.

Remark 4.2. There is an extensive literature dealing with Morrey's notion of Quasiconvexity, [32]. However, from the point of view of mathematical challenges, this concept is not much more than a reformulation of the lower semi-continuity of the energy functionals and as such there remain only technical issues. One needs to develop this idea much further mathematically before it might be usefully applied.

4.3. Nearly conformal deformations. Given a bounded domain $\mathbb{X} \subset \mathbb{R}^{n}$, we look at the mappings $h: \mathbb{X} \rightarrow \mathbb{R}^{n}$ in the reflexive Banach space $\mathfrak{B} \stackrel{\text { def }}{=}$ $\mathscr{W}^{1, n p}\left(\mathbb{X}, \mathbb{R}^{n}\right), \quad 1 \leqslant p<\infty$. Then the following nonlinear functional is well defined on this space.

$$
\mathscr{E}_{n, p}[h]=\int_{\mathbb{X}}\left(|D h(x)|^{n}-n^{n / 2} \operatorname{det} D h(x)\right)^{p} \mathrm{~d} x<\infty
$$

Note that the integrand is non-negative and vanishes only if $h$ satisfies the $n$ dimensional variant of the Cauchy-Riemann system.

$$
\mathcal{K}(D h) \equiv 0, \text { where } \mathcal{K}(X) \stackrel{\text { def }}{=}|X|^{n}-n^{n / 2} \operatorname{det} X \geqslant 0, \text { for } X \in \mathbb{R}^{n \times n}
$$

This motivates our calling $\mathscr{E}_{n, p}$ a nearly conformal energy functional. The Dirichlet boundary value problem consists of minimizing $\mathscr{E}_{n, p}[h]$ subject to mappings $h \in \mathfrak{B}_{\circ} \stackrel{\text { def }}{=} h_{\circ}+\mathscr{W}_{0}^{1, n p}\left(\mathbb{X}, \mathbb{R}^{n}\right)$, where $h_{\circ} \in \mathfrak{B}$ is given boundary data. Here are the essential steps in the Direct Method.

- Coercivity in the mean The reason why the above example is approachable is that while we do not have point-wise coercivity in terms of the integrand, the energy functional $\mathscr{E}_{n, p}$ still exhibits coercivity in the sense of integral means; 
precisely,

$$
\int_{\mathbb{X}}|D h(x)|^{n p} d x \sim \mathscr{E}_{n, p}[h]+\int_{\mathbb{X}}\left|D h_{\circ}(x)\right|^{n p} d x
$$

To see this we appeal to the general (but not very obvious) estimate (10.12) in $[\mathbf{1 9}]$ for mappings $f \in \mathscr{W}_{\circ}^{1, n p}\left(\mathbb{X}, \mathbb{R}^{n}\right)$

$$
\int_{\mathbb{X}}|D f(x)|^{n p} \mathrm{~d} x \sim \mathscr{E}_{n, p}[f]
$$

applied to the mapping $f=h-h_{\circ}$.

- Subgradient estimate This is a fairly direct consequence of polyconvexity of the integrand. Precisely, for $n \times n$-matrices $X, X_{\circ} \in \mathbb{R}^{n \times n}$ we have

$$
\begin{aligned}
\mathcal{K}^{p}(X)-\mathcal{K}^{p}\left(X_{\circ}\right) \geqslant & p \mathcal{K}^{p-1}\left(X_{\circ}\right)\left[\mathcal{K}(X)-\mathcal{K}\left(X_{\circ}\right)\right] \\
\geqslant & n p\left\langle\mathcal{K}^{p-1}\left(X_{\circ}\right)\left|X_{\circ}\right|^{n-2} X_{\circ} \mid X-X_{\circ}\right\rangle \\
& -n^{n / 2} p \mathcal{K}^{p-1}\left(X_{\circ}\right)\left[\operatorname{det} X-\operatorname{det} X_{\circ}\right]
\end{aligned}
$$

- Lower semi-continuity The required inequality (3.3) can be achieved by applying the above subgradient estimate to $X=D f_{i}(x)$ and $X_{\circ}=D f(x)$. We conclude, upon integrating over $\mathbb{X}$, that

$$
\begin{aligned}
\mathscr{E}_{n, p}\left[f_{i}\right]-\mathscr{E}_{n, p}[f] & \\
\geqslant & n p \int_{\mathbb{X}}\left\langle\mathcal{K}^{p-1}(D f)|D f|^{n-2} D f \mid D f_{i}-D f\right\rangle \quad(\text { converging to } 0) \\
& -n^{n / 2} p \int_{\mathbb{X}} \mathcal{K}^{p-1}(D f)\left[\operatorname{det} D f_{i}-\operatorname{det} D f\right] \quad(\text { converging to } 0)
\end{aligned}
$$

The first limit is justified by the fact that $D f_{i} \rightarrow D f$, weakly in the space $\mathscr{L}^{n p}(\mathbb{X})$, and the integration takes place against the factor

$$
\mathcal{K}^{p-1}(D f)|D f|^{n-2} D f \approx|D f|^{n p-1}
$$

which lies in the dual space $\mathscr{L}^{\frac{n p}{n p-1}}(\mathbb{X})$. Similarly, for $p>1$, the null Lagrangians $\operatorname{det} D f_{i}$ converge to $\operatorname{det} D f$ weakly in $\mathscr{L}^{p}(\mathbb{X})$ and we integrate them against a function $\mathcal{K}^{p-1}(D f) \approx|D f|^{n p-n}$ which belongs to the dual space $\mathscr{L}^{\frac{p}{p-1}}(\mathbb{X})$.

The case $p=1$ needs handling with greater care. It is not generally true that $\lim \int_{\mathbb{X}} \operatorname{det} D f_{i}(x) d x=\int_{\mathbb{X}} \operatorname{det} D f(x) d x$, whenever $f_{i} \rightarrow f$, weakly in $\mathscr{W}^{1, n}(\mathbb{X})$. For instance the sequence of Möbius transformations $f_{i}: \mathbb{B} \stackrel{\text { onto }}{\longrightarrow} \mathbb{B}$ of the unit ball $\mathbb{B} \subset \mathbb{R}^{n}$ such that $f_{i}(0) \rightarrow a \in \partial \mathbb{B}$. Their weak limit (indeed locally uniform in $\mathbb{B})$ is $f \equiv a$, which has vanishing Jacobian. However

$$
\int_{\mathbb{B}} \operatorname{det} D f_{i}(x) d x=|\mathbb{B}|>0
$$

The situation is quite different if we confine ourselves to the energy-minimising sequence of mappings $f_{i} \in \mathfrak{B}_{\circ}$ in which $f_{i} \in f_{0}+\mathscr{W}_{0}^{1, n}(\mathbb{X})$. Once the boundary values of $f_{i}$ are fixed, the weak limit enjoys the same boundary 
values; that is, $f \in f_{0}+\mathscr{W}_{0}^{1, n}(\mathbb{X})$. Thus, for all $i=1,2, \ldots$, we have the following identities

$$
\int_{\mathbb{X}} \operatorname{det} D f_{i}(x) \mathrm{d} x=\int_{\mathbb{X}} \operatorname{det} D f_{0}(x) \mathrm{d} x=\int_{\mathbb{X}} \operatorname{det} D f(x) \mathrm{d} x,
$$

by the very definition of null Lagrangians.

Now, with these estimates at hand we may follow the principles of the direct method, and thereby obtain the following.

Proposition 4.3. The nearly conformal energy (4.5), subject to a given boundary data $h_{\circ} \in \mathscr{W}^{1, n p}\left(\mathbb{X}, \mathbb{R}^{n}\right)$, attains its infimum.

The example above shows how the over-arching strategy of the direct method can be nuanced in sophistication at each step. The following specific example further demonstrates this point.

\section{A Very Weak Domain of Definition for Nearly Conformal Deformations}

Given a bounded domain $\mathbb{X} \subset \mathbb{R}^{n}$, we look at the energy functional

$$
\mathscr{E}^{\sharp}[h] \stackrel{\text { def }}{=} \varepsilon\left(\int_{\mathbb{X}}|D h|^{n-1}\right)^{\frac{n}{n-1}}+\int_{\mathbb{X}}\left[\lambda\left|D^{\sharp} h\right|^{\frac{n}{n-1}}-n^{n / 2 n-2} \operatorname{det} D h\right]
$$

with the fixed constant parameters $\varepsilon>0$ and $\lambda>1$. Here the notation $X^{\sharp}$ for $X \in \mathbb{R}^{n \times n}$ stands for the cofactor matrix $X^{\sharp} \in \mathbb{R}^{n \times n}$ whose entries are \pm subdeterminants of size $(n-1) \times(n-1)$ of the matrix $X$. Thus the differential $n$-form $\left[D^{\sharp} h\right] d x$ is a matrix of null Lagrangians. We always have $\left|X^{\sharp}\right|^{\frac{n}{n-1}}$ $n^{n / 2 n-2} \operatorname{det} X \geqslant 0$, equality occurs if and only if $X$ is a similarity matrix of non negative determinant. Thus we always have $\mathscr{E} \sharp[h] \geqslant 0$.

The border line case of $\varepsilon=0$ and $\lambda=1$ reduces to the energy functional which distinguishes orientation preserving conformal mappings as its absolute minima; that is, $\mathscr{E}^{\sharp}[h]=0$. When $n=2$ such minima are either constant mappings, Möbius transformations or a holomorphic function.

From a different perspective energy-minimisers of these type of functionals are discussed in $[\mathbf{3 3}]$, where the existence results are presented for mappings with $\operatorname{det} D h(x) \geqslant 0$, see Lemma 4.1 therein. Let us demonstrate here how to remove this assumption.

At first glance the space $\mathscr{W}^{1, n}\left(\mathbb{X}, \mathbb{R}^{n}\right)$ would seem to be the natural domain of definition of $\mathscr{E} \sharp[h]$. Unfortunately, energy-minimizing sequences need not be bounded in this space. We recall the direct method in this particular case to make a few observations along the way.

\section{- Seek energy-minimisers in the Banach space}

$$
\mathfrak{B} \stackrel{\text { def }}{=} \mathscr{W}^{1, n-1}\left(\mathbb{X}, \mathbb{R}^{n-1}\right)
$$

- $\mathfrak{B} \circ \subset \mathfrak{B}$ This time the subset $\mathfrak{B}_{\circ}$ is somewhat tricky to define and needs handling with care. We have the given boundary data $h \in h_{\circ}+\mathscr{W}_{\circ}^{1, n-1}\left(\mathbb{X}, \mathbb{R}^{n}\right)$. The matrix of cofactors $D^{\sharp} h$ 。 must also lie in the space $\mathscr{L}^{\frac{n}{n-1}}\left(\mathbb{X}, \mathbb{R}^{n \times n}\right)$ just to 
secure the finite energy of $h_{\circ}$. Therefore, we define $\mathfrak{B} \circ \subset \mathfrak{B}$ by the following rule.

$$
\mathfrak{B}_{\circ} \stackrel{\text { def }}{=}\left\{h \in h_{\circ}+\mathscr{W}_{\circ}^{1, n-1}\left(\mathbb{X}, \mathbb{R}^{n}\right): D^{\sharp} h \in \mathscr{L}^{\frac{n}{n-1}}\left(\mathbb{X}, \mathbb{R}^{n \times n}\right)\right\}
$$

Hence $\mathscr{E}^{\sharp}[h]<\infty$ for every $h \in \mathfrak{B}_{\circ}$.

- Coercivity The restriction to mappings $h \in \mathfrak{B}_{\circ}$ also secures a coercivity estimate as follows.

$$
\left(\int_{\mathbb{X}}|D h(x)|^{n-1} d x\right)^{\frac{n}{n-1}}+\int_{\mathbb{X}}\left(\left|D^{\sharp} h(x)\right|^{\frac{n}{n-1}} d x\right) \sim \mathscr{E}^{\sharp}[h]<\infty
$$

Here the implied constant in front of the energy $\mathscr{E}^{\sharp}[h]$ depends only on $\varepsilon>0$ and $\lambda>1$; precisely, it can be shown that it does not exceed $\max \left\{\frac{1}{\varepsilon}, \frac{1}{\lambda-1}\right\}$

- An energy-minimizing sequence. Denoted by $\left\{h_{\kappa}\right\} \subset \mathfrak{B}_{\circ}$ and approaching the infimum energy,

$$
\lim _{\kappa \rightarrow \infty} \mathscr{E}^{\sharp}\left[h_{\kappa}\right]=\inf \left\{\mathscr{E}^{\sharp}[h] ; h \in \mathfrak{B}_{\circ}\right\} \stackrel{\text { def }}{=} \mathrm{E}
$$

Then $h_{\kappa}$ are bounded in the Sobolev space $\mathscr{W}^{1, n-1}\left(\mathbb{X}, \mathbb{R}^{n}\right)$ and $\left\{D^{\sharp} h_{\kappa}\right\}$ are bounded in $\mathscr{L}^{\frac{n}{n-1}}\left(\mathbb{X}, \mathbb{R}^{n \times n}\right)$, both being reflexive Banach spaces. We extract and fix a subsequence, again denoted by $\left\{h_{\kappa}\right\}$, such that:

$-h_{\kappa} \rightarrow h_{\infty}$ weakly in $\mathscr{W}^{1, n-1}\left(\mathbb{X}, \mathbb{R}^{n}\right)$

- $\quad D^{\sharp} h_{\kappa}$ converges weakly in $\mathscr{L}^{\frac{n}{n-1}}\left(\mathbb{X}, \mathbb{R}^{n \times n}\right)$ to a matrix field, say $\mathcal{M} \in$ $\mathscr{L}^{\frac{n}{n-1}}\left(\mathbb{X}, \mathbb{R}^{n \times n}\right)$.

- Note that $D^{\sharp} h_{\kappa} \in \mathscr{L}^{1}\left(\mathbb{X}, \mathbb{R}^{n \times n}\right)$ converge to $D^{\sharp} h_{\infty}$ in the sense of distributions. That is

$$
\int_{\mathbb{X}} \eta(x) D^{\sharp} h_{\kappa}(x) \mathrm{d} x \rightarrow \int_{\mathbb{X}} \eta(x) D^{\sharp} h_{\infty}(x) \mathrm{d} x, \text { for every } \eta \in \mathscr{C}_{0}^{\infty}(\mathbb{X}) \text {. }
$$

This property is referred to in the literature as the weak continuity of Jacobians. Its discovery goes back at least as far as the forgotten paper by R. Caccioppoli [8]. Hence, it is easily seen that $\mathcal{M}(x)=D^{\sharp} h_{\infty}(x)$, almost everywhere.

- Lower semicontinuity. Now a real challenge emerges from trying to evaluate the limit of $\int_{\mathbb{X}} \operatorname{det} D h_{\kappa}(x) \mathrm{d} x$. Although the Jacobians $\operatorname{det} D h_{\kappa}$ remain bounded in $\mathscr{L}^{1}(\mathbb{X})$ it is not generally guaranteed that the integrals $\int_{\mathbb{X}} \operatorname{det} D h_{\kappa}$ admit a subsequence converging to $\int_{\mathbb{X}} \operatorname{det} D h_{\infty}$. This difficulty is to be expected because the gradients $\left\{D h_{\kappa}\right\}$ need not be bounded in $\mathscr{L}^{n}\left(\mathbb{X}, \mathbb{R}^{n \times n}\right)$ (when $n>2$ ).

Remark 5.1. At this point it is worth recalling the concept of distributional Jacobians, defined for mappings $f=\left(f^{1}, f^{2}, \ldots, f^{n}\right)$ of Sobolev class $\mathscr{W}^{1, n-1}\left(\mathbb{X}, \mathbb{R}^{n}\right)$ with $D^{\sharp} f \in \mathscr{L}^{\frac{n}{n-1}}$ as Schwartz distributions

$\Im_{f}[\phi] \stackrel{\text { def }}{=}-\int_{\mathbb{X}} \mathrm{d} f^{1} \wedge \ldots \wedge \mathrm{d} f^{i-1} \wedge f^{i} \mathrm{~d} \phi \wedge \mathrm{d} f^{i+1} \wedge \ldots \wedge \mathrm{d} f^{n}$, for $\phi \in \mathscr{C}_{0}^{\infty}(\mathbb{X})$

It is clear that $\Im_{h_{\kappa}}[\phi] \rightarrow \Im_{h_{\infty}}[\phi]$ for every test function $\phi \in \mathscr{C}_{0}^{\infty}(\mathbb{X})$. Then the classical Stokes theorem reveals that $\Im_{f}[\phi]=\int_{\mathbb{X}} \phi \operatorname{det} D f$, whenever $f \in$ $\mathscr{W}^{1, n}\left(\mathbb{X}, \mathbb{R}^{n}\right)$. But, unfortunately this is not the case with $f=h_{\kappa}$. 
One might hope to circumvent this difficulty by using the idea of biting convergence, first successfully applied to Jacobians by K. Zhang [38]. In fact the above scheme would work if the mappings in question had non-negative Jacobians. We refer again to Lemma 4.1 in [33]. Thus, in essence, the novelty in Example 5 is that we are unconcerned with the orientation of the mappings.

Fortunately the following fact, not so easy to prove, comes to the rescue.

Proposition 5.2. Under the coercivity condition above, the determinants $\operatorname{det} D h_{\kappa}$ are bounded in the Hardy space $\mathscr{H}^{1}(\mathbb{X}) \subset \mathscr{L}^{1}(\mathbb{X})$. As such, they converge to $\operatorname{det} D h_{\infty} \in \mathscr{L}^{1}(\mathbb{X})$ in the sense of distributions,

$$
\int_{\mathbb{X}} \eta(x) \operatorname{det} D h_{\kappa}(x) d x \rightarrow \int_{\mathbb{X}} \eta(x) \operatorname{det} D h_{\infty}(x) d x, \text { for } \eta \in \mathscr{C}_{0}^{\infty}(\mathbb{X})
$$

In fact a stronger statement holds; namely, since the Hardy space $\mathscr{H}^{1}\left(\mathbb{R}^{n}\right)$ is the dual of $V M O\left(\mathbb{R}^{n}\right)$ (functions of vanishing mean oscillation), we conclude that formula (5.3) remains valid for the test functions $\eta \in V M O\left(\mathbb{R}^{n}\right)$ with compact support in $\mathbb{X}$. For a discussion and results concerning biting convergence see $[\mathbf{1 6}]$. Let us demonstrate the lines of reasoning for (5.3) by using the results of [23].

Proof. Suppose we are given a Sobolev mapping $f: \Omega \rightarrow \mathbb{R}^{n}$, defined on a domain $\Omega \subseteq \mathbb{R}^{n}$, whose differential (subdeterminants of size $1 \times 1$ ) and its $(n-1) \times(n-1)$ subdeterminants are integrable with powers $n-1$ and $\frac{n}{n-1}$, respectively,

$$
\left(\int_{\Omega}|D f(x)|^{n-1} d x\right)^{\frac{n}{n-1}}+\int_{\Omega}\left(\left|D^{\sharp} f(x)\right|^{\frac{n}{n-1}} d x\right)<\infty
$$

Then

$$
\int_{\Omega}|\operatorname{det} D f(x)| d x \leqslant\|\operatorname{det} D f\|_{\mathscr{H}^{1}(\Omega)} \leqslant C(n) \int_{\Omega}\left|D^{\sharp} f(x)\right|^{\frac{n}{n-1}} d x
$$

Choose and fix a test function $\eta \in \mathscr{C}_{0}^{\infty}(\mathbb{X})$ and consider the mappings $\eta h_{\kappa}: \mathbb{R}^{n} \rightarrow$ $\mathbb{R}^{n}$ together with their limit $\eta h_{\infty}: \mathbb{R}^{n} \rightarrow \mathbb{R}^{n}$. It is a routine matter to verify that there is a constant $M=M(\eta)<\infty$ such that

$$
\left(\int_{\mathbb{R}^{n}}\left|D\left(\eta h_{\kappa}\right)\right|^{n-1}\right)^{\frac{n}{n-1}}+\int_{\mathbb{R}^{n}}\left|D^{\sharp}\left(\eta h_{\kappa}\right)\right|^{\frac{n}{n-1}} \leqslant M
$$

for all $\kappa=1,2, \ldots$, and also for $\kappa=\infty$. Now Theorem 1.3 in [23] can be used as follows. There is a subsequence, still denoted by $\eta h_{\kappa}$, such that for every $\Phi \in V M O\left(\mathbb{R}^{n}\right)$ it holds that

$$
\int_{\mathbb{R}^{n}} \Phi \operatorname{det}\left[D\left(\eta h_{\kappa}\right)\right] \rightarrow \int_{\mathbb{R}^{n}} \Phi \operatorname{det}\left[D\left(\eta h_{\infty}\right)\right]
$$

The meaning of the integrals is understood in the sense of $\mathscr{H}^{1}-B M O$ duality.

As a test function in $V M O\left(\mathbb{R}^{n}\right)$ we take $\Phi \in \mathscr{C}_{0}^{\infty}\left(\mathbb{R}^{n}\right)$ that is equal to 1 on the support of $\eta$. The integrals in (5.7) exist as Lebesgue integrals and are restricted to the domain $X$, with no factor $\Phi$.

$$
\int_{\mathbb{X}} \operatorname{det}\left[D\left(\eta h_{\kappa}\right)\right] \rightarrow \int_{\mathbb{X}} \operatorname{det}\left[D\left(\eta h_{\infty}\right)\right]
$$

The remainder of the derivation of (5.3) is routine. 
Finally, having Proposition 5.2 in hands, we can argue that $h_{\infty}$ is in fact an energy-minimal deformation for the functional (5.1) in Example 5. A seemingly insignificant fact that the integrand within the square brackets in (5.1) is nonnegative for every map in $\mathfrak{B}_{\circ}$, actually plays a significant role.

Theorem 5.3. The energy functional (5.1) assumes its infimum in $\mathfrak{B}_{\circ}$. In effect, the weak $\mathscr{W}^{1, n-1}$-limit map $h_{\kappa} \rightarrow h_{\infty}$ minimizes the energy.

Proof. We are going to make use of the distributional convergence of the Jacobians as expressed by (5.3). Choose and fix a test function $\eta \in \mathscr{C}_{0}^{\infty}(\mathbb{X})$ such that $0 \leqslant \eta=\eta(x) \leqslant 1$. In addition to (5.3) we have two inequalities due to the lower semicontinuity of the corresponding polyconvex functionals:

(i) $\left(\int_{\mathbb{X}}\left|D h_{\infty}\right|^{n-1}\right)^{\frac{n}{n-1}} \leqslant \liminf _{\kappa \rightarrow \infty}\left(\int_{\mathbb{X}}\left|D h_{\kappa}\right|^{n-1}\right)^{\frac{n}{n-1}}$

(ii) $\int_{\mathbb{X}} \eta(x)\left|D^{\sharp} h_{\infty}(x)\right|^{\frac{n}{n-1}} d x \leqslant \liminf _{\kappa \rightarrow \infty} \int_{\mathbb{X}} \eta(x)\left|D^{\sharp} h_{\kappa}(x)\right|^{\frac{n}{n-1}} d x$

(iii) $\quad \int_{\mathbb{X}} \eta(x) \operatorname{det} D h_{\infty}(x) d x=\lim _{\kappa \rightarrow \infty} \int_{\mathbb{X}} \eta(x) \operatorname{det} D h_{\kappa}(x) d x$

Adding these terms together yields

$$
\begin{aligned}
& \mathscr{A}_{\eta}\left[h_{\infty}\right] \\
& \stackrel{\text { def }}{=} \varepsilon\left(\int_{\mathbb{X}}\left|D h_{\infty}\right|^{n-1}\right)^{\frac{n}{n-1}}+\int_{\mathbb{X}} \eta\left[\lambda\left|D^{\sharp} h_{\infty}\right|^{\frac{n}{n-1}}-n^{n / n-2} \operatorname{det} D h_{\infty}\right] \\
& \leqslant \liminf _{\kappa \rightarrow \infty}\left\{\varepsilon\left(\int_{\mathbb{X}}\left|D h_{\kappa}\right|^{n-1}\right)^{\frac{n}{n-1}}+\int_{\mathbb{X}} \eta\left[\lambda\left|D^{\sharp} h_{\kappa}\right|^{\frac{n}{n-1}}-n^{n / n-2} \operatorname{det} D h_{\kappa}\right]\right\} \\
& \leqslant \liminf _{\kappa \rightarrow \infty}\left\{\varepsilon\left(\int_{\mathbb{X}}\left|D h_{\kappa}\right|^{n-1}\right)^{\frac{n}{n-1}}+\int_{\mathbb{X}}\left[\lambda\left|D^{\sharp} h_{\kappa}\right|^{\frac{n}{n-1}}-n^{n / n-2} \operatorname{det} D h_{\kappa}\right]\right\} \\
& =\liminf _{\kappa \rightarrow \infty} \mathscr{E}^{\sharp}\left[h_{\kappa}\right]=\mathrm{E} \quad \text { ( a quantity independent of } 0 \leqslant \eta=\eta(x) \leqslant 1 \text { ). }
\end{aligned}
$$

In the first line of the above inequalities, the integrand within square brackets is nonnegative and $\mathscr{L}^{1}$-integrable. Therefore, by letting $\eta$ approach $\equiv 1$ point-wise, we may (and do) pass to the supremum. This gives us the desired estimate

$$
\mathscr{E}^{\sharp}\left[h_{\infty}\right]=\mathscr{A}_{\equiv 1}\left[h_{\infty}\right] \leqslant \mathrm{E} \text { (the infimum energy) }
$$

Remark 5.4. It seems likely that Theorem 5.3 remains valid for $\lambda=1$ and $\varepsilon>0$ as well. To gain this, however, one must devise a mean coercivity (as opposed to point-wise coercivity) for the energy integrals of $\left|D^{\sharp} h\right|^{\frac{n}{n-1}}$; say, by analogy with (4.7).

The borderline case of $\varepsilon=0$ and $\lambda=1$ (that is very weak conformality) seems to be of interest for further studies.

\section{Free Lagrangians and Frictionless Deformations.}

It is of great interest to study the relationship between topology and analysis in the setting of extremal problems. In particular one is often interested in obtaining a 
"nice" mapping in the homotopy class of a given mapping between spaces. After the Riemann mapping theorem perhaps the best known example of this are questions around the existence of harmonic mappings in a homotopy class of mappings. This problem was pioneered in the work of Eells and Sampson from 1964 [14], covered in the later long papers of Eels and Lemaire $[\mathbf{1 2}, \mathbf{1 3}]$ and has since found wide application in very many areas of mathematics, far too numerous to note. To formalise this problem in the setting of this article we come to the notion of a free Lagrangian.

Let us say to begin with that free Lagrangians are quite like null Lagrangians, and we represent them by the symbol

$$
\mathscr{F}_{L}(\mathbb{X}, \mathbb{Y})
$$

It has to be emphasised that this notation refers to a given pair of domains $\mathbb{X}, \mathbb{Y} \subset$ $\mathbb{R}^{n}$ of the same topological type. That is they are homeomorphic. As opposed to the situation considered for null Lagrangians, the concept of free Lagrangians concerns nonlinear differential $n$-forms $\mathbf{L}(x, h, D h) d x$, defined for Sobolev homeomorphisms $h: \mathbb{X} \stackrel{\text { onto }}{\longrightarrow} \mathbb{Y}$, whose integral means (which we usually call the energy of $h$ ) depend only on the homotopy class of $h$, and not on its boundary values. This of course leads to frictionless type problems.

Definition 6.1. Suppose that a given energy integral

$$
\mathscr{F}[h] \stackrel{\text { def }}{=} \int_{\mathbb{X}} \mathbf{F}(x, h, D h) d x, \text { where } \quad \mathbf{F}: \mathbb{X} \times \mathbb{Y} \times \mathbb{R}^{n \times n} \rightarrow \mathbb{R}
$$

converges for all Sobolev mappings of class $\mathscr{W}^{1, p}(\mathbb{X}, \mathbb{Y})$. We say that the differential $n$-form $\mathbf{F}(\cdot, \cdot, \cdot) d x$ is a Free Lagrangian if

$$
\mathscr{F}\left[h_{1}\right]=\mathscr{F}\left[h_{2}\right], \text { whenever } h_{1} \simeq h_{2}
$$

That is, whenever the Sobolev homeomorphisms $h_{1}, h_{2}: \mathbb{X} \stackrel{\text { onto }}{\longrightarrow} \mathbb{Y}$, in $\mathscr{W}^{1, p}\left(\mathbb{X}, \mathbb{R}^{n}\right)$, are homotopy equivalent.

It is important to note here that we are only considering homotopy equivalence between surjections (sometimes perhaps with other restrictions such as homeomorphisms). For instance if $\mathbb{X}, \mathbb{Y}$ are topological balls, then any continuous map $\mathbb{X} \rightarrow \mathbb{Y}$ is certainly homotopic to a constant map and little more can be said.

We shall now make this concept clear with selected examples and illustrate how this leads to questions of the existence of frictionless energy minimal deformations.

\subsection{Examples.}

$\mathscr{F}_{L}^{1}$ ) (A volume form in $\mathbb{X}$ ) This simple example is still useful (as we will see) and is given by an integrand independent of $h$ :

$$
\mathbf{F}(x, y, \xi) d x=\mathbf{F}(x) d x, \text { where } \mathbf{F} \in \mathscr{L}^{1}(\mathbb{X})
$$

$\left.\mathscr{F}_{L}^{2}\right)$ (A pullback of a volume form in $\mathbb{Y}$ )

An example, to some extent dual to the above, is the pullback of a volume form $\Phi(y) d y$ with $\Phi \in \mathscr{L}^{1}(\mathbb{Y})$,

$$
\mathbf{F}(x, y, \xi) d x=\Phi(y) \operatorname{det} \xi d x
$$


We note the identity

$$
\mathscr{F}[h]=\int_{\mathbb{X}} \Phi(h) J(x, h) d x=\int_{\mathbb{Y}} \Phi(y) \mathrm{d} y
$$

which holds for all orientation preserving homeomorphisms $h: \mathbb{X} \stackrel{\text { onto }}{\longrightarrow} \mathbb{Y}$ in the Sobolev space $\mathscr{W}^{1, n}(\mathbb{X}, \mathbb{Y})$. In other words, using the notation of exterior algebra, the $n$-form $\Phi(h) d h^{1} \wedge \ldots \wedge d h^{n}$ is a free Lagrangian. Applications are still limited because the associated energies do not recognise the geometric shape of the domains $\mathbb{X}$ and $\mathbb{Y}$ only their volumes. A myriad of differential expressions, representing free Lagrangians, are yet to be found. The next two examples (again dual to each other) provide very useful free Lagrangians for a pair of round annuli

$$
\mathbb{X}=\mathbb{A}=\{x: \quad r<|x|<R\}, \quad \text { and }, \quad \mathbb{Y}=\mathbb{A}^{*}=\left\{y: \quad r_{*}<|y|<R_{*}\right\}
$$

$\left.\mathscr{F}_{L}^{3}\right)$ (Radial increment of $|h| ; \mathscr{F}_{L}\left(\mathbb{A}, \mathbb{A}^{*}\right)$ )

Proposition 6.2. The following differential $n$-form $\mathbf{F}(x, h, D h) \mathrm{d} x$, :

$$
\frac{(d|h|) \wedge \star d|x|}{|h||x|^{n-1}} \stackrel{\text { def }}{=} \sum_{i=1}^{n} \frac{x_{i} d x_{1} \wedge \ldots \wedge d x_{i-1} \wedge d|h| \wedge d x_{i+1} \wedge \ldots \wedge d x_{n}}{|h||x|^{n}}
$$

is a free Lagrangian in the homotopy class of orientation preserving homeomorphisms that preserve the order of the boundary components of the annuli $\mathbb{A}$ and $\mathbb{A}^{*}$. Precisely, for such homeomorphisms of Sobolev class $\mathscr{W}^{1,1}\left(\mathbb{A}, \mathbb{A}^{*}\right)$, we have

$$
\int_{\mathbb{A}} \mathbf{F}(x, h, D h) \mathrm{d} x=\int_{\mathbb{A}} \frac{d|h| \wedge \star d|x|}{|h||x|^{n-1}}=\operatorname{Mod} \mathbb{A}^{*} \stackrel{\text { def }}{=} \log \frac{R^{*}}{r^{*}}
$$

Here we have used the Hodge star duality operator in the exterior algebra; namely, $*: \Lambda^{1}\left(\mathbb{R}^{n}\right) \stackrel{\text { onto }}{\longrightarrow} \Lambda^{n-1}\left(\mathbb{R}^{n}\right)$.

Observe that the function $|h|: \mathbb{A} \rightarrow\left(r_{*}, R_{*}\right)$ extends continuously to the closure of $\mathbb{A}$. That $h$ preserves the order of the spherical boundary components simply means that $|h(x)|=r_{*}$ for $|x|=r$ and $|h(x)|=R_{*}$ for $|x|=R$. Accordingly,

$$
\mathbf{F} d x=\frac{d|h| \wedge \star d|x|}{|h||x|^{n-1}} \in \mathscr{F}_{L}\left(\mathbb{A}, \mathbb{A}^{*}\right)
$$

$\left.\mathscr{F}_{L}^{4}\right)$ (Spherical derivatives of $h ; \mathscr{F}_{L}\left(\mathbb{A}, \mathbb{A}^{*}\right)$ )

Another free Lagrangian in $\mathbf{F}(\cdot, \cdot, \cdot) \in \mathscr{F}_{L}\left(\mathbb{A}, \mathbb{A}^{*}\right)$, dual to that in Proposition 6.2 , exploits topological degree of the mappings $h: \mathbb{S}_{t}^{n-1} \rightarrow \mathbb{R}^{n} \backslash\{0\}$ restricted to the concentric spheres of radii $t \in(r, R)$. The degree is equal to 1 on every sphere, which yields

Proposition 6.3. The following differential $n$-form $\mathbf{F}(x, h, D h) \mathrm{d} x$ :

$$
\frac{d|x|}{|x|} \wedge h^{\sharp} \omega=\sum_{i=1}^{n} \frac{h^{i} d h^{1} \wedge \ldots \wedge d h^{i-1} \wedge d|x| \wedge d h^{i+1} \wedge \ldots \wedge d h^{n}}{|x||h|^{n}}
$$

is a free Lagrangian in the class of all orientation preserving homeomorphisms $h \in \mathscr{W}^{1, n-1}\left(\mathbb{A}, \mathbb{A}^{*}\right)$. Precisely, we have

$$
\int_{\mathbb{A}} \mathbf{F}(x, h, D h) \mathrm{d} x=\int_{\mathbb{A}} \frac{d|x|}{|x|} \wedge h^{\sharp} \omega=\operatorname{Mod} \mathbb{A} \stackrel{\text { def }}{=} \log \frac{R}{r}
$$


Here $h^{\sharp} \omega$ stands for the pullback of the $(n-1)$-area form defined in $\mathbb{A}^{*} ;$ namely,

$$
\omega(y)=\sum_{i=1}^{n}(-1)^{i} \frac{y^{i} d y^{1} \wedge \ldots \wedge d y^{i-1} \wedge d y^{i+1} \wedge \ldots \wedge d y^{n}}{|y|^{n}}
$$

This is none other than the $(n-1)$ area form on any $(n-1)$-closed surface in $\mathbb{A}^{*}$ that is homologous to $\mathbb{S}^{n-1}$. A far more detailed exposition, suited to this example, is presented in [24, Chapters 6 and 7].

6.2. The Nitsche frictionless problem. Given a pair planar annuli $\mathbb{A}=\{x \in$ $\mathbb{C}: r<|x|<R\}$ and $\mathbb{A}^{*}=\left\{y \in \mathbb{C}: r_{*}<|y|<R_{*}\right\}$, the objective is to minimize the Dirichlet energy subject to Sobolev homeomorphisms $h: \mathbb{A} \stackrel{\text { onto }}{\longrightarrow} \mathbb{A}^{*}$ in $\mathscr{W}^{1,2}(\mathbb{A}, \mathbb{C})$.

$$
\mathscr{E}_{2}[h]=\int_{\mathbb{A}}|D h(x)|^{2} \mathrm{~d} x
$$

Note that no boundary values of these homeomorphisms are prescribed, whence the name frictionless is given to this problem. We denote this class of mappings by $\mathscr{H}^{1,2}\left(\mathbb{A}, \mathbb{A}^{*}\right)$.

Naturally, polar coordinates

$$
x=t e^{i \theta}, \quad r<t<R \text { and } 0 \leqslant \theta<2 \pi
$$

are best suited. The radial (normal) and angular (tangential) derivatives of $h$ are defined by

$$
h_{N}(x)=\frac{\partial h\left(t e^{i \theta}\right)}{\partial t}, \quad t=|x|
$$

and

$$
h_{T}(x)=\frac{1}{t} \frac{\partial h\left(t e^{i \theta}\right)}{\partial \theta}, \quad t=|x|
$$

The stored energy integrand takes the form

$$
|D h(x)|^{2}=\left|h_{N}(x)\right|^{2}+\left|h_{T}(x)\right|^{2}
$$

This also provides an effective formula for the Jacobian determinant

$$
J_{h}(x)=\operatorname{det} \operatorname{Dh}(x)=\operatorname{Im}\left(\overline{h_{N}} h_{T}\right) \leqslant\left|h_{N}\right|\left|h_{T}\right| .
$$

Our free Lagrangians $\left.\left.\mathscr{F}_{L}^{1}\right), \ldots, \mathscr{F}_{L}^{4}\right)$ in $\mathscr{F}_{L}\left(\mathbb{A}, \mathbb{A}^{*}\right)$ can easily be stated using polar coordinates in even slightly greater generality. The main players and their energy integrals for $h \in \mathscr{H}^{1,2}\left(\mathbb{A}, \mathbb{A}^{*}\right)$ are:

$$
\int_{\mathbb{A}} M(x) d x, \quad M \in \mathscr{L}^{1}(\mathbb{A})
$$

$\left.\mathscr{F}_{2}\right) \quad \int_{\mathbb{A}} N(|h|) J_{h}(x) \mathrm{d} x=2 \pi \int_{r_{*}}^{R_{*}} N(s) s \mathrm{~d} s$

$$
\int_{\mathbb{A}} A(|h|) \frac{|h|_{N}}{|x|} \mathrm{d} x=2 \pi \int_{r_{*}}^{R_{*}} A(s) \mathrm{d} s \quad A \in \mathscr{L}^{1}\left(r_{*}, R_{*}\right)
$$

$\left.\mathscr{F}_{4}\right) \quad \int_{\mathbb{A}} B(|x|) \operatorname{Im} \frac{h_{T}}{h} \mathrm{~d} x=2 \pi \int_{r}^{R} B(t) d t, \quad B \in \mathscr{L}^{1}(r, R)$. 
6.3. Energy minimisers among radial mappings. It is natural to first look at the radial mappings as candidates for energy-minimisers. However, this expectation is far from being guaranteed. In spite of the radial symmetry of the annuli and the invariance of the Dirichlet energy under rotations of $\mathbb{A}$ and $\mathbb{A}^{*}$, such a lack of symmetry of the energy-minimisers has, quite surprisingly, been confirmed already in the analogous Nitsche problem in dimensions $n \geqslant 3$. Nevertheless, the extremals within the radial mappings give us the pinpoint of free-Lagrangian to solve the minimization problem in full generality. The general form of a radial mapping is as follows.

$$
h_{\circ}(x)=H(|x|) \frac{x}{|x|}, \quad H:[r, R] \stackrel{\text { onto }}{\longrightarrow}\left[r_{*}, R_{*}\right] \text { is continuously increasing }
$$

If one seeks harmonic radial mappings then

$$
\Delta h_{\circ}(x)=h_{t t}+\frac{1}{t} h_{t}+t^{-2} h_{\theta \theta}=0,
$$

where $x=t e^{i \theta}$. Then $H=H(t)$ must satisfy the Euler's ordinary differential equation

$$
t^{2} \ddot{H}(t)+t \dot{H}(t)-H(t)=0 \text { for } r<t<R
$$

Its two fundamental solutions $t$ and $\frac{1}{t}$ generate all solutions

$$
H(t)=a t+b / t
$$

To ensure the boundary constraints, $H(r)=r_{*}$ and $H(R)=R_{*}$, we must set,

$$
H(t)=a t+b / t, \text { where } a=\frac{R R_{*}-r r_{*}}{R^{2}-r^{2}} \text { and } b=\frac{R^{2} r r_{*}-r^{2} R R_{*}}{R^{2}-r^{2}}
$$

It is advantageous to transfer the above equation to the first order ODE by simply multiplying by the integrating factor $-2 \dot{H}(t)$. We obtain the characteristic equation

$$
\mathcal{L}[H] \stackrel{\text { def }}{=} H^{2}-t^{2} \dot{H}^{2} \equiv c, \text { where } c \in \mathbb{R} \text { is a constant. }
$$

Note that, as opposed to the second order Laplace equation, the first order characteristic equation admits an additional solution, namely, a constant function.

We shall be concerned with monotone $\mathscr{C}^{1}$-solutions $H:[r, R] \stackrel{\text { onto }}{\longrightarrow}\left[r_{*}, R_{*}\right]$, thus having $\dot{H}(t) \geqslant 0$. This includes solutions that are partially constant. In particular, they may squeeze but not fold subintervals. Under these assumptions the respective radial mappings $h_{\circ}$ become uniform limits of homeomorphisms, as desired in the weak formulation of the principle of noninterpenetration of matter discussed earlier.

Remark 6.4. Equation (6.14), for such solutions, is none other than the variational equation of the minimization problem when confined to the radial mappings. This fact, though natural to expect, is not automatic and we will exploit it.

We will not use the explicit formulas (6.13), but only the characteristic equation (6.14). This means that our arguments have to be developed for frictionless problems where one can identify the PDEs for the energy-minimisers, if not their explicit solutions. 
There is a useful quantity associated with the radial mappings called the elasticity of stretching

$$
\eta_{H}(t) \stackrel{\text { def }}{=} \frac{t \dot{H}(t)}{H(t)}
$$

All $\mathscr{C}^{1}$-solutions fall into three categories. If $c$ is the constant at (6.14) we say that:

- $H$ is conformal if $c=0$, equivalently $\eta_{H}(t)=1$ iff $\frac{R_{*}}{r_{*}}=\frac{R}{r}$

- $H$ is expanding if $c<0$, equivalently $\eta_{H}(t)>1$ iff $\frac{R_{*}}{r_{*}}>\frac{R}{r}$

- $H$ is contracting if $c>0$, equivalently $\eta_{H}(t)<1$ iff $\frac{R_{*}}{r_{*}}<\frac{R}{r}$

The characteristic equation (6.14) has an injective solution if and only if

$$
\frac{1}{2}\left(\frac{R}{r}+\frac{r}{R}\right) \leqslant \frac{R_{*}}{r_{*}}
$$

We call the set of values for whiich (6.15) holds the Nitsche range, it includes the expanding case. We reserve the notation $F(\tau) \stackrel{\text { def }}{=} H^{-1}(\tau)$ for $r_{*}<\tau<R_{*}$. Thus the characteristic equation reads as

$$
\left(\frac{F}{\dot{F}}\right)^{2}-\tau^{2} \equiv c \quad F(\tau)=\frac{\tau+\sqrt{\tau^{2}-c}}{2 a} .
$$

When the reverse inequality to $(6.15)$ holds, we say the data lies beyond the Nitsche range. We consider a $\mathscr{C}^{1,1}$-solution $H:[r, R] \stackrel{\text { onto }}{\longrightarrow}\left[r_{*}, R_{*}\right]$ of $(6.14)$ defined by the rule.

$$
H(t)=\left\{\begin{array}{ll}
r_{*} & t \in[r, \rho] \\
H_{\rho}(t) & t \in[\rho, R]
\end{array}, \quad \rho \text { is determined by } \quad \frac{1}{2}\left(\frac{R}{\rho}+\frac{\rho}{R}\right)=\frac{R_{*}}{r_{*}}\right.
$$

Here , $H_{\rho}(t)=\frac{r_{*}}{2}\left(t+\frac{\rho}{t}\right)$, is an increasing solution $H_{\rho}:[\rho, R] \stackrel{\text { onto }}{\longrightarrow}\left[r_{*}, R_{*}\right]$, of the characteristic equation (6.14).

6.4. The conformal case. In this case the pullback of the area form alone is sufficient to identify the energy-minimal mappings. Precisely, using $\mathscr{F}_{2}$ ), we obtain.

$$
\int_{\mathbb{A}}|D h(x)|^{2} \mathrm{~d} x=\int_{\mathbb{A}}\left|h_{N}\right|^{2}+\left|h_{T}\right|^{2} \geqslant 2 \int_{\mathbb{A}}\left|h_{N}\right|\left|h_{T}\right| \geqslant 2 \int_{\mathbb{A}} J_{h}(x) d x=2\left|\mathbb{A}^{*}\right|
$$

Equality occurs only for a similarity transformation of $\mathbb{A}$ onto $\mathbb{A}^{*}$ (scalar multiple of a rotation).

6.5. The expanding case. Choose and fix a radial mapping $h_{\circ}=H(|x|) \frac{x}{|x|}$, where $H$ solves Equation (6.14) with $c<0$. Explicitly, in complex notation, we have the formula

$$
h_{\circ}(z)=a z+\frac{b}{\bar{z}} .
$$

It is true that $h_{\circ}$ turns out to be the energy-minimal solution among radial mappings, but we shall not exploit this property; equation (6.14) is sufficient. Now suppose we are given an arbitrary mapping $h \in \mathscr{H}^{1,2}\left(\mathbb{A}, \mathbb{A}^{*}\right)$. We shall derive a series of sharp estimates involving $h$ and $D h$, each of which becomes an equality 
if $h=h_{\circ}$ - the radial Nitche map. Let us introduce the following functions; first defined for $r_{*} \leqslant \tau \leqslant R_{*}$ by the rule

$$
p(\tau)=\eta_{F}(\tau)=\frac{\tau \dot{F}(\tau)}{F(\tau)}<1, \quad\left(p(\tau)=\frac{\tau \sqrt{\tau^{2}-c}+\tau^{2}}{\tau \sqrt{\tau^{2}-c}+\tau^{2}-c}\right)
$$

We note that $p(|h(x)|)=\frac{\left|h_{N}(x)\right|}{\left|h_{T}(x)\right|}$ for $h \stackrel{\text { def }}{=} h_{\circ}$. The second function of two variables is defined by

$$
A(t, \tau) \stackrel{\text { def }}{=} \frac{F(\tau)}{t \dot{F}(\tau)}, \quad \text { for } r \leqslant t \leqslant R \text { and } r_{*} \leqslant \tau \leqslant R_{*}
$$

We note that the function $x \mapsto A(|x|,|h(x)|)$ is equal to $|h|_{N}(x)$ in the case $h=$ $h_{\circ}(x)$.

To proceed we develop algebraic inequalities which lead to the lower bounds of the integrand by means of free-Lagrangians. In our case, the following point-wise inequality holds whenever $0 \leqslant p \leqslant 1$ and $A \geqslant 0$.

$$
\begin{aligned}
|D h|^{2} & =\left|h_{N}\right|^{2}+\left|h_{T}\right|^{2} \geqslant\left(1-p^{2}\right)\left|h_{N}\right|^{2}+2 p\left|h_{N}\right|\left|h_{T}\right| \\
& \geqslant\left(1-p^{2}\right) 2 A|h|_{N}-\left(1-p^{2}\right) A^{2}+2 p J_{h}
\end{aligned}
$$

Now, according to $(6.16)$ we have $\left(1-p^{2}\right) A^{2}=c|x|^{-2}$ and so

$$
|D h|^{2} \geqslant 2\left(\frac{F(|h|)}{\dot{F}(|h|)}-\frac{|h|^{2} \dot{F}(|h|)}{F(|h|)}\right) \frac{|h|_{N}}{|x|}-\frac{c}{|x|^{2}}+2 p(|h|) J_{h}
$$

Here the right hand side consists of free-Lagrangians and equality occurs for the radial Nitsche map $h_{\circ}$. Hence

$$
\int_{\mathbb{A}}|D h(x)|^{2} d x \geqslant \int_{\mathbb{A}}\left|D h_{\circ}(x)\right|^{2} d x, \text { as desired. }
$$

6.6. The contracting case. In contrast to the expanding case, in the contracting case apply spherical free-Lagrangians $\mathscr{F}_{4}$ ) in place of the radial free-Lagrangian $\left.\mathscr{F}_{3}\right)$. We discuss two subcases.

6.6.1. Annuli still within the Nitsche range at (6.15). In this subcase the radial solution $h_{\circ}$ still remains injective. The first step is to choose a good point-wise inequality. Precisely, for all parameters $0 \leqslant q \leqslant 1$ and $B \geqslant 0$ it holds, as is easily verified, that

$$
\begin{aligned}
|D h|^{2} & =\left|h_{N}\right|^{2}+\left|h_{T}\right|^{2} \geqslant\left(1-q^{2}\right)\left|h_{T}\right|^{2}+2 q\left|h_{N}\right|\left|h_{T}\right| \\
& \geqslant\left(1-q^{2}\right) 2 B\left|h_{T}\right|-\left(1-q^{2}\right) B^{2}+2 q J_{h} \\
& \geqslant\left(1-q^{2}\right) 2 B|h| \operatorname{Im} \frac{h_{T}}{h}-\left(1-q^{2}\right) B^{2}+2 q J_{h}
\end{aligned}
$$

Then, in the second step, we take for $q$ and $B$ the following functions

$$
\begin{aligned}
q & =q(|h|)=\frac{1}{\eta_{F}(|h|)}=\frac{F(|h|)}{|h| \dot{F}(|h|)}<1 \\
B & =B(|x|,|h|)=\left(\frac{H^{2}(|x|)}{|x|}-|x| \dot{H}^{2}(|x|)\right) \frac{1}{|h|\left(1-q^{2}(|h|)\right)} .
\end{aligned}
$$


Now since $|h|^{2}\left(1-q^{2}(|h|) \equiv c\right.$ by (6.16) we obtain the desired lower bound on $|D h|^{2}$ by free-Lagrangians.

$$
\begin{aligned}
|D h|^{2} \geqslant 2 & \left.\frac{H^{2}(|x|)}{|x|}-|x| \dot{H}^{2}(|x|)\right) \operatorname{Im} \frac{h_{T}}{h} \\
& -\frac{1}{c}\left(\frac{H^{2}(|x|)}{|x|}-|x| \dot{H}^{2}(|x|)\right)^{2}+\frac{2 J_{h}}{\eta_{F}(|h|)}
\end{aligned}
$$

Here again the right hand side consists of free-Lagrangians and equality occurs for the radial Nitsche map $h_{\circ}$. Hence

$$
\int_{\mathbb{A}}|D h(x)|^{2} d x \geqslant \int_{\mathbb{A}}\left|D h_{\circ}(x)\right|^{2} d x
$$

6.6.2. Annuli beyond the Nitsche bound. This means that

$$
\frac{1}{2}\left(\frac{R}{r}+\frac{r}{R}\right)>\frac{R_{*}}{r_{*}}
$$

It is exactly in this subcase that the radial solution $h_{\circ}$ fails to be injective. A plausible candidate for the energy-minimal deformation is the squeezing/stretching radial mapping $h_{\circ}(x)=H(|x|) \frac{x}{|x|}$, where $H:[r, R] \stackrel{\text { onto }}{\longrightarrow}\left[r_{*}, R_{*}\right]$ is given by (6.17). For $\rho<|x|<R$ we apply (6.22) and obtain a lower bound in terms of freeLagrangians

$|D h|^{2} \geqslant 2\left(\frac{H_{\rho}^{2}(|x|)}{|x|}-|x| \dot{H}_{\rho}^{2}(|x|)\right) \operatorname{Im} \frac{h_{T}}{h}-\frac{1}{c}\left(\frac{H_{\rho}^{2}(|x|)}{|x|}-|x| \dot{H}_{\rho}^{2}(|x|)\right)^{2}+2 \frac{J_{h}}{\eta_{F_{\rho}}(|h|)}$

The remaining free-Lagrangians lower bound for $r<|x| \leqslant \rho$ is just as easy to verify.

$$
|D h|^{2}=\left|h_{N}\right|^{2}+\left|h_{T}\right|^{2} \geqslant 2 B r_{*} \operatorname{Im} \frac{h_{T}}{h}-B^{2} \text { with } B(|x|)=r_{*}|x|^{-1}
$$

The point is that both lower bounds also become equalities for $h_{\circ}$. In conclusion,

$$
\int_{\mathbb{A}}|D h(x)|^{2} d x \geqslant \int_{\mathbb{A}}\left|D h_{\circ}(x)\right|^{2} d x, \quad \text { as well }
$$

All the above cases summarize as follows

Theorem 6.5. The frictionless deformations $h: \mathbb{A} \stackrel{\text { onto }}{\longrightarrow} \mathbb{A}^{*}$ between annuli assume their minimum Dirichlet energy among radial mappings.

Actually, our proofs easily give an even more precise statement; namely, the energy-minimal mappings are unique up to a rotation of the variable $x \in \mathbb{A}$ or, equivalently, a rotation of $y \in \mathbb{A}^{*}$.

\section{Mappings of $\mathscr{L}^{p}$-Minimal Distortion}

In relation to the previous problem, we have already noted in the introduction at (2.6) and the preamble, that should $h$ be a homeomorphism of Sobolev class, then

$$
\int_{\mathbb{A}}|D h|^{2} d z=\int_{\mathbb{A}^{*}} \mathbb{K}(z, f) d z, \quad f=h^{-1}: \mathbb{A}^{*} \rightarrow \mathbb{A} .
$$


If $\Phi: V \stackrel{\text { onto }}{\longrightarrow} W$ is a conformal mapping between planar domains, and $f: U \stackrel{\text { onto }}{\longrightarrow}$ $V$ is a homeomorphism of Sobolev class $\mathscr{W}^{1, p}(U, V), p \geq 1$, then it is an elementary calculation that

$$
\mathbb{K}(z, \Phi \circ f)=\mathbb{K}(z, f)
$$

and hence

$$
\int_{U} \mathbb{K}(z, \Phi \circ f) d z=\int_{U} \mathbb{K}(z, f) d z
$$

Let $\Omega$ be a doubly connected planar domain. The Riemann mapping theorem for doubly connected domains tells us that there is a single conformal invariant, the modulus of $\Omega$ - denoted $\bmod (\Omega)$, which identifies a conformally equivalent round annulus $\mathbb{A}=\mathbb{A}(r, R)$ and $\log R / r=\bmod (\mathbb{A})=\bmod (\Omega)$. We can now restate the results of $\S 6.2$ as follows.

Theorem $7.1(\mathbf{p}=\mathbf{1})$. Let $\Omega$ be a doubly connected planar domain with $s=\bmod (\Omega)$ and $\mathbb{A}=\mathbb{A}(1, R), r=\bmod (\mathbb{A})$. Then there is a homeomorphism $f: \mathbb{A} \stackrel{\text { onto }}{\longrightarrow} \Omega$ of Sobolev class $\mathscr{W}^{1,1}(\mathbb{A}, \Omega)$ minimising $\int_{\mathbb{A}} \mathbb{K}(z, f) d z$ if and only if $\cosh (s) \leq e^{r}$. This minimiser is a diffeomorphism and is unique up to conformal automorphisms of $\Omega$.

This is the Nitsche bound (6.15). There some subtlety here regarding the regularity of $h=f^{-1}$. In particular is $h \in \mathscr{W}^{1,2}(\Omega, \mathbb{A})$ ? This is discussed in $[\mathbf{1}, \S 21]$ and [17]. Despite being of considerable interest, we set aside discussion of these issues in this article. In contrast to Theorem 7.1 we have the following theorem concerning extremal quasiconformal mappings.

Theorem $7.2(\mathbf{p}=\infty)$. Let $\Omega$ be a doubly connected planar domain with $0<$ $\bmod (\Omega)<\infty$. Then for every $0<\bmod (\mathbb{A})<\infty$, there is a homeomorphism $f$ : $\mathbb{A} \stackrel{\text { onto }}{\longrightarrow} \Omega$ of Sobolev class $\mathscr{W}^{1,2}(\mathbb{A}, \Omega)$ minimising $\|\mathbb{K}(z, f)\|_{L^{\infty}(\mathbb{A})}$ This minimiser is a diffeomorphism and is unique up to conformal automorphisms of $\Omega$.

One can reduce this to the case $\Omega=\mathbb{A}\left(1, e^{s}\right)$ using (7.2) and $\mathbb{A}=\mathbb{A}\left(1, e^{r}\right)$. Then identify the extremal quasiconformal mapping as the radial map $z \mapsto z|z|^{\alpha-1}$, $\alpha=\log (s) / \log (r)$.

There is a surprising difference here between Theorem 7.1 and Theorem 7.2. Namely there is always an extremal quasiconformal mapping, but a minimiser of mean distortion exists only within a range of moduli - precisely the Nitsche range. This leads naturally to the question of what happens for $1<p<\infty$ when we minimise the functional

$$
f \mapsto \int_{\mathbb{A}} \mathbb{K}^{p}(z, f) d z
$$

or perhaps the more general case of including a positive weight $\eta: \mathbb{A} \rightarrow(0, \infty)$ to obtain the functional

$$
f \mapsto \int_{\mathbb{A}} \mathbb{K}^{p}(z, f) \eta(z) d z
$$

The answer is a little surprising and proved in [30], (this also was recently developed in the context of free-Lagrangians in [25] by the first and third authors, but here we give a different, but actually equivalent, approach). Further, the result suggests an interesting critical phase type phenomenon. When $p<1$, apart from the identity map, minimisers never exist. When $p=1$ we observe Nitsche type phenomenon; minimisers exist within a range of conformal moduli determined by properties of 
the weight function and not otherwise. When $p>1$ minimisers always exist. We will now go through the proof of this.

7.1. Mappings of finite distortion. A homeomorphism $f: \Omega \rightarrow \Omega^{\prime}$ between planar domains of Sobolev class $\mathscr{W}_{l o c}^{1,1}\left(\Omega, \Omega^{\prime}\right)$ has finite distortion if the Jacobian determinant $J(z, f)$ is nonnegative and there is a function $\mathbb{K}(z, f)$ finite almost everywhere such that

$$
|D f(z)|^{2} \leqslant \mathbb{K}(z, f) J(z, f) .
$$

Recall that $\mathbb{K}(z, f)$ is the distortion of the mapping $f$. We saw earlier at (2.6) that $\mathbb{K}$ is a measure of the anisotropic local stretching.

Mappings of finite distortion are generalisations of quasiconformal homeomorphisms and have found considerable recent application in geometric function theory and nonlinear PDEs, [1]. We define annuli

$$
\mathbb{A}_{1}=\{1 \leqslant|z| \leqslant R\}, \quad \mathbb{A}_{2}=\{1 \leqslant|z| \leqslant S\}
$$

with moduli $\sigma_{1}=\log (R)$ and $\sigma_{2}=\log (S)$. We consider homeomorphisms of finite distortion $f: \mathbb{A}_{1} \rightarrow \mathbb{A}_{2}$ mapping the boundary components to each other,

$$
f(\{|z|=1\})=\{|z|=1\}, \quad \text { and } \quad f(\{|z|=R\})=\{|z|=S\} .
$$

On the annulus $\mathbb{A}_{1}$ place a positive weight $\eta: \mathbb{A}_{1} \rightarrow \mathbb{R}_{+}$. In polar coordinates

$$
f_{z}=\frac{1}{2} e^{-i \theta}\left(f_{\rho}-\frac{i}{\rho} f_{\theta}\right), \quad f_{\bar{z}}=\frac{1}{2} e^{i \theta}\left(f_{\rho}+\frac{i}{\rho} f_{\theta}\right)
$$

and $\left|f_{z}\right|^{2}+\left|f_{\bar{z}}\right|^{2}=\frac{1}{2}\left(\left|f_{\rho}\right|^{2}+\rho^{-2}\left|f_{\theta}\right|^{2}\right), J(z, f)=\left|f_{z}\right|^{2}-\left|f_{\bar{z}}\right|^{2}=\frac{1}{\rho} \operatorname{Im}\left(f_{\theta} \overline{f_{\rho}}\right)$ which together yield

$$
\mathbb{K}(z, f)=\frac{\left|f_{z}\right|^{2}+\left|f_{\bar{z}}\right|^{2}}{\left|f_{z}\right|^{2}-\left|f_{\bar{z}}\right|^{2}}=\frac{\rho\left|f_{\rho}\right|^{2}+\rho^{-1}\left|f_{\theta}\right|^{2}}{2 \operatorname{Im}\left(f_{\theta} \overline{f_{\rho}}\right)} .
$$

Given a convex function $\varphi:[1, \infty) \rightarrow[0, \infty)$ a Nitsche type problem asks us to establish the existence or otherwise of a minimiser (or perhaps stationary point) of the functional

$$
f \mapsto \int_{\mathbb{A}_{1}} \varphi(\mathbb{K}(z, f)) \eta(z)|d z|^{2} .
$$

Thus we seek a deformation of the annulus $\mathbb{A}_{1}$ to $\mathbb{A}_{2}$ which minimises some weighted $L^{\varphi}$ average of the distortion. $\S 6.2$ deals with the case $\varphi(t)=t$ and $\eta(x) \equiv 1$; minimisers of mean distortion.

7.2. Grötzsch type problems. The classical Grötzsch problem asks one to identify the linear mapping as the homeomorphism of least maximal distortion between two rectangles. Put

$$
\mathbf{Q}_{1}=[0, \ell] \times[0,1], \quad \mathbf{Q}_{2}=[0, L] \times[0,1]
$$

and suppose we have a deformation of finite distortion $f: \mathbf{Q}_{1} \rightarrow \mathbf{Q}_{2}$ with

$$
\operatorname{Re} f(0, y)=0, \quad \operatorname{Re} f(\ell, y)=L, \quad \operatorname{Im} f(x, 0)=0, \quad \operatorname{Im} f(x, 1)=1
$$

(so $f$ is orientation preserving and maps edges to edges). This Sobolev map is absolutely continuous on lines and so $\int_{0}^{\ell} \operatorname{Re}\left(f_{x}\right) d x=L$ and $\int_{0}^{1} \operatorname{Im}\left(f_{y}\right) d y=1$ for almost all $y$ and $x$ respectively, and hence

$$
\operatorname{Re} \int_{\mathbf{Q}_{1}} f_{x}(z)|d z|^{2}=L, \quad \operatorname{Im} \int_{\mathbf{Q}_{1}} f_{y}(z)|d z|^{2}=\ell .
$$


The distortion function is

$$
\mathbb{K}(z, f)=\frac{\left|f_{x}\right|^{2}+\left|f_{y}\right|^{2}}{J(z, f)} \geqslant 1
$$

A Grötzsch problem seeks a minimiser, satisfying the boundary conditions (7.7), to the functional

$$
f \mapsto \int_{\mathbf{Q}_{1}} \varphi(\mathbb{K}(z, f)) \lambda(z)|d z|^{2}
$$

for some positive weight function $\lambda$.

7.3. Equivalence between Nitsche and Grötzsch problems. The universal cover of an annulus is effected by the exponential map, so $z \mapsto \exp (2 \pi z)$ takes $z=x+i y \in[0, L] \times[0,1]$ to $\mathbb{A}_{2}$ if $\sigma_{2}=\log (S)=2 \pi L$. A branch of logarithm must be chosen to define an "inverse" map $[0, \ell] \times[0,1] \rightarrow \mathbb{A}_{1}$. If $f: \mathbb{A}_{1} \rightarrow \mathbb{A}_{2}$ is given, then we can define $\tilde{f}(z)=\frac{1}{2 \pi} \log (f(\exp 2 \pi z))$. A particular point here is that $\log$ is conformal (in fact we only really need log to define a univalent conformal mapping from $\mathbb{A}_{1} \backslash([1, S] \times\{0\})$ to $\mathbf{Q}_{2}$ with edges matching up) so

$$
\mathbb{K}(z, \tilde{f})=\mathbb{K}\left(z, \frac{1}{2 \pi} \log \left(f\left(e^{2 \pi z}\right)\right)\right)=\mathbb{K}\left(z, f\left(e^{2 \pi z}\right)\right),
$$

and hence a change of variables yields

$$
\begin{aligned}
\int_{\mathbf{Q}_{1}} \varphi(\mathbb{K}(z, \tilde{f})) \lambda(z)|d z|^{2} & =\int_{\mathbf{Q}_{1}} \varphi\left(\mathbb{K}\left(z, f\left(e^{2 \pi z}\right)\right) \lambda(z)|d z|^{2}\right. \\
& =4 \pi^{2} \int_{\mathbb{A}_{1}} \varphi(\mathbb{K}(w, f)) \lambda(z) e^{-4 \pi \operatorname{Re}(z)}|d w|^{2} .
\end{aligned}
$$

With the choice

$$
\eta(w)=4 \pi^{2} \lambda(z) e^{-4 \pi \operatorname{Re}(z)}, \quad e^{z}=w
$$

the equivalence between the two problems (with related weight) is seen. Again, the exact branch of log here will be immaterial to our considerations.

Remark 7.3. In fact the equivalence between Nitsche and Grötzsch problems is only when one assumes periodic boundary behaviour in the Grötzsch problem. We will be fortunate in that the absolute minimisers for the Grötzsch problem in the situations we consider do exhibit this periodicity and so can be lifted.

7.4. Sublinear distortion functionals; $p<1$. The purpose of this brief section is to show that minimisers never exist for the $L^{p}$-minimisation problem when $p<1$. We recall from [3, Theorem 5.3] (actually the proof of this result) the following.

Lemma 7.4. Let $\Psi(t)$ be a positive strictly increasing function of sublinear growth:

$$
\lim _{t \rightarrow \infty} \frac{\Psi(t)}{t}=0
$$

Let $\mathbb{B}=\mathbb{D}\left(z_{0}, r\right)$ be a round disk and suppose that $f_{0}: \mathbb{B} \rightarrow \mathbb{C}$ is a homeomorphism of finite distortion with $\int_{\mathbb{B}} \Psi\left(\mathbb{K}\left(z, f_{0}\right)\right)<\infty$. Then there is a sequence of mappings of finite distortion $f_{n}: \mathbb{B} \rightarrow f_{0}(\mathbb{B})$ with $f_{n}(\zeta)=f_{0}(\zeta)$ near $\partial \mathbb{B}$ and with

- $\mathbb{K}\left(z, f_{n}\right) \rightarrow 1$ uniformly on compact subsets of $\mathbb{B}$

- $\int_{\mathbb{B}} \Psi\left(\mathbb{K}\left(z, f_{n}\right)\right) \rightarrow \int_{\mathbb{B}} \Psi(1)$ as $n \rightarrow \infty$.

We now prove the following theorem. 
Theorem 7.5. Let $\Psi(t)$ be a positive strictly increasing function of sublinear growth, let $\Omega$ be a domain and let $\lambda(z) \in L^{\infty}(\Omega)$ be a positive weight. Suppose that $g_{0}: \Omega \rightarrow \mathbb{C}$ is a homeomorphism of finite distortion with

$$
\int_{\Omega} \Psi\left(\mathbb{K}\left(z, g_{0}\right)\right)<\infty
$$

Then there is a sequence of mappings of finite distortion $g_{n}: \Omega \rightarrow g_{0}(\Omega)$ with $g_{n}(\zeta)=g_{0}(\zeta), \zeta \in \partial \Omega$ with

$$
\int_{\Omega} \Psi\left(\mathbb{K}\left(z, g_{n}\right)\right) \lambda(z) \rightarrow \Psi(1) \int_{\Omega} \lambda(z) \quad \text { as } n \rightarrow \infty
$$

Proof. Let $\epsilon>0$. Since $\left(\Psi\left(\mathbb{K}\left(z, g_{0}\right)\right)-\Psi(1)\right) \lambda(z) \in L^{1}(\Omega)$ we can choose a finite collection of disjoint disks contained in $\Omega$, say $\left\{B_{i}\right\}_{i=1}^{N}$, so that

$$
\left|\int_{\Omega \backslash \cup B_{i}}\left(\Psi\left(\mathbb{K}\left(z, g_{0}\right)\right)-\Psi(1)\right) \lambda(z) d z\right|<\epsilon / 2 .
$$

Next, for each $i$ we use Lemma 7.4 in the obvious way to find $h_{i}: B_{i} \rightarrow \mathbb{C}$ with $h_{i}=g_{0}$ in a neighbourhood of $\partial B_{i}$ and

$$
\left|\int_{B_{i}} \Psi\left(\mathbb{K}\left(z, h_{i}\right)\right) \lambda(z)-\Psi(1) \int_{B_{i}} \lambda(z)\right|<\frac{\epsilon}{2 N}
$$

Then the map

$$
g_{\epsilon}(z)= \begin{cases}g_{0}(z) & z \in \Omega \backslash \bigcup B_{i} \\ h_{i}(z) & z \in B_{i}\end{cases}
$$

is of finite distortion and

$$
\begin{aligned}
\left|\int_{\Omega} \Psi\left(\mathbb{K}\left(z, g_{\epsilon}\right)\right) \lambda(z)-\Psi(1) \int_{\Omega} \lambda(z)\right|= & \mid \int_{\Omega \backslash \bigcup B_{i}}\left(\Psi\left(\mathbb{K}\left(z, g_{\epsilon}\right)\right)-\Psi(1)\right) \lambda(z) d z \\
& +\sum_{i=1}^{N} \int_{B_{i}}\left(\Psi\left(\mathbb{K}\left(z, h_{i}\right)\right)-\Psi(1)\right) \lambda(z) d z \mid \\
& <\epsilon
\end{aligned}
$$

The result follows.

And the next corollary is what we seek.

Corollary 7.6. Let $\Psi(t)$ be a positive strictly increasing function of sublinear growth, let $\Omega$ be a domain and let $\lambda(z) \in L^{\infty}(\Omega)$ be a positive weight. Suppose that $g_{0}: \Omega \rightarrow \mathbb{C}$ is a homeomorphism of finite distortion with

$$
\int_{\Omega} \Psi\left(\mathbb{K}\left(z, g_{0}\right)\right)<\infty
$$

Then

$$
\min _{\mathcal{F}} \int_{\Omega} \Psi(\mathbb{K}(z, g)) \lambda(z)=\Psi(1) \int_{\Omega} \lambda(z) d z
$$

with equality achieved by a mapping of finite distortion if and only if the boundary values of $g_{0}$ are shared by a conformal mapping. Here $\mathcal{F}$ consists of homeomorphisms of finite distortion $g$ with $g \mid \partial \Omega=g_{0}$. 
7.5. Minimisers of convex distortion functionals. A natural class of homeomorphic mappings between rectangles satisfying the Grötzsch boundary conditions (7.7) are those of the form

$$
f_{0}(z)=u(x)+i y
$$

which will correspond to the lifts of the radial stretchings. For these mappings we have $\left(f_{0}\right)_{x}=u_{x}$ and $\left(f_{0}\right)_{y}=i$. We will show these mappings are the extremals for our mapping problems, but we will have to deal with degenerate situations as well - in particular where $f_{0}$ is not well defined, but has a well defined inverse. These are topologically monotone mappings we have talked about earlier as local uniform limits of homeomorphisms, and so for us as limits of minimising sequences. In order to avoid excess technical complications we make the following assumptions:

Let $w=a+i b \in[0, L] \times[0,1]$ and set

$$
g_{0}(w)=v(a)+i b
$$

where $v:[0, L] \rightarrow[0, \ell]$ is an absolutely continuous, increasing (but not necessarily strictly increasing) surjection. The derivative $v_{a}$ of $v$ is a non-negative $\mathscr{L}^{1}([0, \ell])$ function which if it is positive almost everywhere makes $v$ strictly increasing and we may set

$$
f_{0}=g_{0}^{-1}:[0, \ell] \times[0,1] \rightarrow[0, L] \times[0,1]
$$

We now proceed as follows.

Lemma 7.7. Set $g_{0}(w)=v(a)+i b$, where $v:\left[0, L_{0}\right] \rightarrow[0, \ell]$ is an absolutely continuous, increasing surjection. Let $f:[0, \ell] \times[0,1] \rightarrow[0, L] \times[0,1]$ be a homeomorphism of finite distortion satisfying the boundary conditions (7.7). Then

$$
\left|v_{a}(a) f_{x}\left(g_{0}(w)\right)+i f_{y}\left(g_{0}(w)\right)\right|^{2} \geqslant 0
$$

Equality holds for $f$ and almost every $w$ if and only if $v$ is strictly increasing $L=L_{0}$ and $f=g_{0}^{-1}$.

Proof. We consider

$$
h(w)=\left(f \circ g_{0}\right)(w)
$$

The mapping $h \in \mathscr{W}_{l o c}^{1,1}$ and maps $\left[0, L_{0}\right] \times[0,1] \rightarrow[0, L] \times[0,1]$ respecting the sides. We compute the $\bar{w}$-derivative of $h$;

$$
\begin{aligned}
2 h_{\bar{w}}(w)= & f_{z}(v(a)+i b) v_{a}(a)+f_{\bar{z}}(v(a)+i b) v_{a}(a) \\
& +i f_{z}(v(a)+i b)-i f_{\bar{z}}(v(a)+i b) \\
= & f_{x}\left(g_{0}(w)\right) v_{a}(a)+i f_{y}\left(g_{0}(w)\right)=0
\end{aligned}
$$

as an $\mathscr{L}^{1}$-function. Thus $h$ is analytic by the Looman-Menchoff theorem. The boundary conditions and analyticity imply that $h$ is a homeomorphism of the boundary which must therefore be a homeomorphism of the rectangles. Then $L=L_{0}$ and $h$ must be the identity since the two rectangles have moduli $L_{0}$ and $L$. The result follows

For a suitable function $v$ as above, let us write $z=x+i y$ where

$$
z=g_{0}(w) \text { and } \omega(x)=v_{a}(a) .
$$


We note that $\omega$ is well defined. First, $g_{0}$ is a surjection and if $g_{0}\left(w_{1}\right)=g_{0}\left(w_{2}\right)$, then $w_{1}$ and $w_{2}$ lie in a common interval on which $v$ is constant, whereupon $v_{a}\left(a_{1}\right)=$ $v_{a}\left(a_{2}\right)=0$. However if $\omega(x)>0$, then

$$
\left|\omega(x) f_{x}(z)+i f_{y}(z)\right|^{2} \geqslant 0
$$

with equality almost everywhere if and only if $g_{0}$ is a homeomorphism and $f_{0}=g_{0}^{-1}$. Also, when $\omega>0, v$ is strictly increasing,

$$
g_{0}^{-1}(z)=f_{0}(z)=u(x)+i y, \quad \omega(x)=1 / u_{x}(x) .
$$

We now suppose that $\omega>0$ and expand out (7.20).

$$
\begin{aligned}
0 & \leqslant\left|\omega(x) f_{x}+i f_{y}\right|^{2}=\left(\omega(x) f_{x}+i f_{y}\right)\left(\omega(x) \overline{f_{x}}-i \overline{f_{y}}\right) \\
& =\omega^{2}(x)\left|f_{x}\right|^{2}+\left|f_{y}\right|^{2}-2 \operatorname{Im}\left(\omega(x) f_{y} \overline{f_{x}}\right)
\end{aligned}
$$

which yields

$$
\omega^{2}(x)\left|f_{x}\right|^{2}+\left|f_{y}\right|^{2} \geqslant 2 \omega(x) \operatorname{Im}\left(f_{y} \overline{f_{x}}\right) .
$$

Notice that if we write $f=U+i V$, then

$$
\operatorname{Im}\left(f_{y} \overline{f_{x}}\right)=\operatorname{Im}\left(U_{x}(z)-i V_{x}(z)\right)\left(U_{y}(z)+i V_{y}(z)\right)=J(z, f),
$$

so (7.22) gives us

$$
\omega^{2}(x)\left|f_{x}\right|^{2}+\left|f_{y}\right|^{2} \geqslant 2 \omega(x) J(z, f)
$$

with equality almost everywhere if and only if $f=f_{0}$ (with the implication that $f_{0}$ is a homeomorphism). We can rewrite (7.23) in two different ways. Namely

$$
\begin{aligned}
& \left|f_{x}\right|^{2}+\left|f_{y}\right|^{2} \geqslant\left(1-\omega^{-2}(x)\right)\left|f_{y}\right|^{2}+2 \omega^{-1}(x) J(z, f), \\
& \left|f_{x}\right|^{2}+\left|f_{y}\right|^{2} \geqslant\left(1-\omega^{2}(x)\right)\left|f_{x}\right|^{2}+2 \omega(x) J(z, f),
\end{aligned}
$$

which gives us two estimates on the distortion function (writing $J=J(z, f)$ ),

$$
\begin{aligned}
& \mathbb{K}(z, f) \geqslant\left(1-\omega^{-2}(x)\right) \frac{\left|f_{y}\right|^{2}}{J}+2 \omega^{-1}(x), \\
& \mathbb{K}(z, f) \geqslant\left(1-\omega^{2}(x)\right) \frac{\left|f_{x}\right|^{2}}{J}+2 \omega(x) .
\end{aligned}
$$

Next, when $\omega>0$ almost everywhere we can define $f_{0}$ by (8.1) with (??). Then

$$
\begin{aligned}
& \mathbb{K}\left(z, f_{0}\right)=\left(1-\omega^{-2}(x)\right) \frac{\left|\left(f_{0}\right)_{y}\right|^{2}}{J_{0}}+2 \omega^{-1}(x), \\
& \mathbb{K}\left(z, f_{0}\right)=\left(1-\omega^{2}(x)\right) \frac{\left|\left(f_{0}\right)_{x}\right|^{2}}{J_{0}}+2 \omega(x),
\end{aligned}
$$

and thus we have our first useful inequalities

Lemma 7.8. If $\omega(x)>0$, then

$$
\mathbb{K}(z, f)-\mathbb{K}\left(z, f_{0}\right) \geqslant\left(1-\omega^{-2}(x)\right)\left[\frac{\left|f_{y}\right|^{2}}{J}-\frac{\left|\left(f_{0}\right)_{y}\right|^{2}}{J_{0}}\right],
$$

and

$$
\mathbb{K}(z, f)-\mathbb{K}\left(z, f_{0}\right) \geqslant\left(1-\omega^{2}(x)\right)\left[\frac{\left|f_{x}\right|^{2}}{J}-\frac{\left|\left(f_{0}\right)_{x}\right|^{2}}{J_{0}}\right]
$$

with equality holding almost everywhere in either inequality if and only if $f=f_{0}$. 
We leave it to the reader to establish the elementary inequality for complex numbers $X, X_{0}$ and real $J, J_{0}$,

$$
\frac{|X|^{2}}{J}-\frac{\left|X_{0}\right|^{2}}{J_{0}} \geqslant 2 \operatorname{Re}\left(\frac{\overline{X_{0}}}{J_{0}}\left(X-X_{0}\right)\right)-\frac{\left|X_{0}\right|^{2}}{J_{0}^{2}}\left(J-J_{0}\right),
$$

with equality holding if and only if $X / X_{0}=J / J_{0}$ is a positive real number (expand $\left.\left|X / X_{0}-J / J_{0}\right|^{2} \geqslant 0\right)$. This shows

$$
(X, Y, J) \mapsto \frac{|X|^{2}+|Y|^{2}}{J}
$$

to be convex on $\mathbb{C} \times \mathbb{C} \times \mathbb{R}_{+}$. We apply (7.26) and this requires that the coefficient $\left(1-\omega^{-2}(x)\right)>0$ in the first case or $\left(1-\omega^{2}(x)\right)>0$ in the second. Since this depends on $u_{x}$ for the candidate extremal mapping, we carry along the two inequalities and write $\mathbb{K}_{0}=\mathbb{K}\left(z, f_{0}\right)$. First note that if $\varphi: \mathbb{R} \rightarrow \mathbb{R}$ is convex, then its graph lies above any tangent line:

$$
\varphi(\mathbb{K})-\varphi\left(\mathbb{K}_{0}\right) \geqslant \varphi^{\prime}\left(\mathbb{K}_{0}\right)\left(\mathbb{K}-\mathbb{K}_{0}\right) .
$$

Notice that if $\varphi^{\prime \prime}>0$, equality holds here if and only if $\mathbb{K}=\mathbb{K}_{0}$. This therefore yields the following two inequalities:

$$
\begin{aligned}
\varphi(\mathbb{K}(z, f))-\varphi\left(\mathbb{K}\left(z, f_{0}\right)\right) \geqslant & \left(1-\omega^{-2}(x)\right) \varphi^{\prime}\left(\mathbb{K}_{0}\right) \\
& {\left[2 \operatorname{Re}\left(\frac{\overline{\left(f_{0}\right)_{y}}}{J_{0}}\left(f_{y}-\left(f_{0}\right)_{y}\right)\right)-\frac{\left|\left(f_{0}\right)_{y}\right|^{2}}{J_{0}^{2}}\left(J-J_{0}\right)\right], } \\
\varphi(\mathbb{K}(z, f))-\varphi\left(\mathbb{K}\left(z, f_{0}\right)\right) \geqslant & \left(1-\omega^{2}(x)\right) \varphi^{\prime}\left(\mathbb{K}_{0}\right) \\
& {\left[2 \operatorname{Re}\left(\frac{\overline{\left(f_{0}\right)_{x}}}{J_{0}}\left(f_{x}-\left(f_{0}\right)_{x}\right)\right)-\frac{\left|\left(f_{0}\right)_{x}\right|^{2}}{J_{0}^{2}}\left(J-J_{0}\right)\right] . }
\end{aligned}
$$

Now $\left(f_{0}\right)_{y}=i$ and $\left(f_{0}\right)_{x}=1 / \omega(x)=J_{0}$ so these equations read as

$$
\begin{aligned}
& \varphi(\mathbb{K}(z, f))-\varphi\left(\mathbb{K}\left(z, f_{0}\right)\right) \\
& \geqslant\left(1-\frac{1}{\omega^{2}(x)}\right) \varphi^{\prime}\left(\mathbb{K}_{0}\right)\left[\frac{2}{J_{0}} \operatorname{Im}\left(f_{y}-1\right)-\frac{J-J_{0}}{J_{0}^{2}}\right] \\
&= 2\left(\omega(x)-\frac{1}{\omega(x)}\right) \varphi^{\prime}\left(\mathbb{K}_{0}\right) \operatorname{Im}\left(f_{y}-1\right) \\
&+\left(\omega^{2}(x)-1\right) \varphi^{\prime}\left(\mathbb{K}_{0}\right)\left(J_{0}-J\right), \\
& \varphi(\mathbb{K}(z, f))-\varphi\left(\mathbb{K}\left(z, f_{0}\right)\right) \\
& \geqslant\left(1-\omega^{2}(x)\right) \varphi^{\prime}\left(\mathbb{K}_{0}\right)\left[2 \operatorname{Re}\left(f_{x}-\left(f_{0}\right)_{x}\right)-\left(J-J_{0}\right)\right] .
\end{aligned}
$$

Now we want to multiply these two inequalities by a weight function $\lambda(x)$ and integrate. We are naturally led to consider the Euler-Lagrange equation for the variational problem minimising

$$
\int_{\mathbf{Q}} \varphi(\mathbb{K}(z, f)) \lambda(x)|d z|^{2}
$$


among functions of the form (7.15). This equation reduces to the next equation in one real variable

$$
\frac{d}{d x}\left[\lambda(x)\left(1-\frac{1}{u_{x}^{2}}\right) \varphi^{\prime}\left(u_{x}+\frac{1}{u_{x}}\right)\right]=0
$$

We would therefore like $\omega(x)$ to be chosen so that

$$
\lambda(x)\left(1-\omega^{2}(x)\right) \varphi^{\prime}\left(\omega(x)+\frac{1}{\omega(x)}\right)=\alpha \neq 0
$$

for a real constant $\alpha$. This equation implicitly defines $\omega$ directly, and does not involve any of its derivatives.

Remark 7.9. We postpone a discussion of boundary values for the solution $f_{0}$ (really $\left.g_{0}\right)$ that we seek. Set

$$
\int_{0}^{\ell} \frac{d x}{\omega(x)}=L_{0}
$$

The boundary conditions we want are that $L=L_{0}$ to identify the minimum. However, if $L_{0}<L$, then Lemma 7.7 still applies - and we obtain strict inequality. Also, we note that from (7.30), with an assumption that $\lambda>0$ and $\varphi^{\prime}$ are continuous, that $\omega=0$ implies that $\lambda(x) \varphi^{\prime}(\infty)=\alpha$. In particular, we cannot have $\omega(x)=0$ unless $\varphi^{\prime}$ is bounded - a condition we will see again.

We now suppose that we have (7.30) holding almost everywhere and $L_{0}<L$. Then (7.30) forces $0 \leq \omega(x)<1$ for all $x$ or $\omega(x)>1$ for all $x$. The case $\omega \equiv 1$, implies that $\alpha=0$ and hence $g_{0}=f_{0}=i d e n t i t y$. The first case (where we will ultimately have to deal with degeneration as we cannot guarantee the boundary conditions) has $u_{x}>1$ and so must correspond to stretching $L>\ell$. In the other case $\ell<L$.

We proceed as follows.

$$
\begin{aligned}
\int_{\mathbf{Q}_{1}} \varphi(\mathbb{K}(z, f)) \lambda(x)|d z|^{2} & \geqslant \int_{\mathbf{Q}_{1}} \varphi\left(\mathbb{K}\left(z, f_{0}\right)\right) \lambda(x)|d z|^{2}-\alpha \int_{\mathbf{Q}_{1}}\left(J_{0}-J\right)|d z|^{2} \\
+ & 2 \int_{\mathbf{Q}_{1}} \lambda(x)\left(\omega(x)-\frac{1}{\omega(x)}\right) \varphi^{\prime}\left(\mathbb{K}_{0}\right) \operatorname{Im}\left(f_{y}-1\right)|d z|^{2}, \\
\int_{\mathbf{Q}_{1}} \varphi(\mathbb{K}(z, f)) \lambda(x)|d z|^{2} & \geqslant \int_{\mathbf{Q}_{1}} \varphi\left(\mathbb{K}\left(z, f_{0}\right)\right) \lambda(x)|d z|^{2}+\alpha \int_{\mathbf{Q}_{1}}\left(J_{0}-J\right)|d z|^{2} \\
& +2 \alpha \int_{\mathbf{Q}_{1}} \operatorname{Re}\left(f_{x}-\left(f_{0}\right)_{x}\right)|d z|^{2} .
\end{aligned}
$$

For an arbitrary Sobolev homeomorphism it is well known that

$$
\int_{\mathbf{Q}_{1}} J|d z|^{2} \leqslant\left|\mathbf{Q}_{2}\right|=L=\int_{0}^{\ell} u_{x}(x) d x=\int_{\mathbf{Q}_{1}} J_{0}|d z|^{2}
$$

We will use the first inequality above when $\alpha<0$ and the second when $\alpha>0$. Thus, for $\alpha<0$

$$
\begin{aligned}
\int_{\mathbf{Q}_{1}} \varphi(\mathbb{K}(z, f)) \lambda(x)|d z|^{2} \\
\quad \geqslant \int_{\mathbf{Q}_{1}} \varphi\left(\mathbb{K}\left(z, f_{0}\right)\right) \lambda(x)|d z|^{2}+2 \int_{\mathbf{Q}_{1}} \lambda\left(\omega-\frac{1}{\omega}\right) \varphi^{\prime}\left(\mathbb{K}_{0}\right) \operatorname{Im}\left(f_{y}-1\right)|d z|^{2},
\end{aligned}
$$


while for $\alpha>0$ we have

$$
\begin{aligned}
\int_{\mathbf{Q}_{1}} \varphi(\mathbb{K}(z, f)) \lambda(x)|d z|^{2} \\
\quad \geqslant \int_{\mathbf{Q}_{1}} \varphi\left(\mathbb{K}\left(z, f_{0}\right)\right) \lambda(x)|d z|^{2}+2 \alpha \int_{\mathbf{Q}_{1}} \operatorname{Re}\left(f_{x}-\left(f_{0}\right)_{x}\right)|d z|^{2}
\end{aligned}
$$

Next, from (7.8) we see that

$$
\begin{aligned}
\int_{\mathbf{Q}_{1}} \lambda(x)\left(\omega(x)-\frac{1}{\omega(x)}\right) \varphi^{\prime}\left(\mathbb{K}_{0}\right) \operatorname{Im}\left(f_{y}-1\right)|d z|^{2} \\
\quad=\int_{0}^{\ell} \lambda(x)\left(\omega(x)-\frac{1}{\omega(x)}\right) \varphi^{\prime}\left(\mathbb{K}_{0}\right)\left[\int_{0}^{1} \operatorname{Im}\left(f_{y}-1\right) d y\right] d x=0
\end{aligned}
$$

and

$$
\int_{\mathbf{Q}_{1}} \operatorname{Re}\left(f_{x}-\left(f_{0}\right)_{x}\right)|d z|^{2}=\int_{0}^{1}\left[\int_{0}^{\ell} \operatorname{Re}\left(f_{x}-\left(f_{0}\right)_{x}\right) d x\right] d y=0 .
$$

Thus we have established

Theorem 7.10. Let $\lambda(x)>0$ be a positive weight and $\varphi:[1, \infty) \rightarrow[0, \infty)$ be convex increasing. Let the function $u:[0, \ell] \rightarrow[0, L]$

$$
u(0)=0, \quad u(\ell)=L_{0} \leq L
$$

be a solution to the ordinary differential equation

$$
\lambda(x)\left(1-\frac{1}{u_{x}^{2}(x)}\right) \varphi^{\prime}\left(u_{x}(x)+\frac{1}{u_{x}(x)}\right)=\alpha
$$

where $\alpha$ is a nonzero constant. Set

$$
f_{0}(z)=u(x)+i y, \quad f_{0}:[0, \ell] \times[0,1] \rightarrow\left[0, L_{0}\right] \times[0,1] .
$$

Let $f:[0, \ell] \times[0,1] \rightarrow[0, L] \times[0,1]$ be a surjective homeomorphism of finite distortion with

$$
\operatorname{Re} f(0, y)=0, \quad \operatorname{Re} f(\ell, y)=L, \quad \operatorname{Im} f(x, 0)=0, \quad \operatorname{Im} f(x, 1)=1
$$

Then

$$
\int_{\mathbf{Q}_{1}} \varphi(\mathbb{K}(z, f)) \lambda(x)|d z|^{2} \geqslant \int_{\mathbf{Q}_{1}} \varphi\left(\mathbb{K}\left(z, f_{0}\right)\right) \lambda(x)|d z|^{2} .
$$

Equality holds if and only if $f=f_{0}$. In particular, if $L_{0}<L$, then this inequality is strict.

Notice $\alpha=0$ gives the identity mapping - clearly always an absolute minimiser when it is a candidate.

7.6. Degenerate cases. Theorem 7.10 identifies the extremal homeomorphism of finite distortion when we can find $\alpha$ so that $L_{0}=L$. We will see later that this is not always possible and then Theorem 7.10 provides us with the unattainable lower bound $\int_{\mathbf{Q}_{1}} \varphi\left(\mathbb{K}\left(z, f_{0}\right)\right) \lambda(x)$ - since the inequality is strict. When $L_{0}<L$ of course $f_{0}$ is not a candidate mapping for the minimisation problem - so it might not be surprising the bound is unattainable. However it might be possible that this value is the limit of a minimising sequence of candidates. What we want to do here is to find circumstances in which this happens. 
Theorem 7.11. Suppose that $f_{0}$ is defined as in Theorem 7.10 above and for no choice of $\alpha$ is it possible that $L=L_{0}$. Suppose that $\varphi^{\prime}$ is bounded. Then there is a sequence of surjective homeomorphism of finite distortion $f_{j}:[0, \ell] \times[0,1] \rightarrow$ $[0, L] \times[0,1]$ such that

$$
\int_{\mathbf{Q}_{1}} \varphi\left(\mathbb{K}\left(z, f_{j}\right)\right) \lambda(x)|d z|^{2}=\int_{\mathbf{Q}_{1}} \varphi\left(\mathbb{K}\left(z, f_{0}\right)\right) \lambda(x)|d z|^{2}
$$

In particular, under these circumstances there is no extremal homeomorphism of finite distortion whatsoever.

Remark. We will see in the next few sections the condition $\phi^{\prime}$ bounded is necessary for nonexistence of minimisers, but not sufficient. The behaviour of the weight $\lambda$ near its minimum determines whether we can solve the boundary problem for arbitrary $L$.

Proof. Our assumption is that $\varphi$ is convex increasing and thus $\varphi^{\prime}$ is positive and increasing, not necessarily strictly. We may also assume $\lim _{t \rightarrow \infty} \varphi^{\prime}(t)=1$. The function $t \mapsto\left(1-t^{-2}\right) \varphi^{\prime}(t+1 / t)$ is strictly increasing and our solution $u^{\alpha}$ is obtained by the rule $u_{x}^{\alpha}(x)=t_{x}$ where $\left(1-t_{x}^{-2}\right) \varphi^{\prime}\left(t_{x}+1 / t_{x}\right)=\alpha / \lambda(x)$. This implies that $\alpha \leq \alpha_{0}=\min _{x} \lambda(x)$. It is easy to see that $u^{\alpha}(\ell) \nearrow u^{\alpha_{0}}(\ell)$ and our hypothesis is that last value is $L_{0}<L$. Thus for $\alpha \leq \alpha_{0}$, the family $u^{\alpha} \in \mathscr{W}^{1,1}([0, \ell])$, with a uniform bound. Further $u_{0}=u^{\alpha_{0}}$ is strictly increasing with derivative tending to $\infty$ as $x$ approaches a minimum, say $x_{0}$, of $\lambda$ (which may be an endpoint of $[0, \ell]$ ). Let

$$
g_{0}(w)=v_{0}(a)+i b, \quad v=u_{0}^{-1}
$$

Then $\left(v_{0}\right)_{a}(a)=1 /\left(u_{0}\right)_{x}(x)$ with $u_{0}(x)=a \in\left[0, L_{0}\right]$. With $u_{0}\left(x_{0}\right)=a_{0}$ we have $\left(v_{0}\right)_{a}\left(a_{0}\right)=0$. We now define a new function $g:[0, L] \times[0,1] \rightarrow[0, \ell] \times[0,1]$ by simply defining $g(w)=v(a)+i b$ to be constant near $x_{0}$. That is (with appropriate modification should $x_{0}$, the minimum of $\lambda$ be an endpoint)

$$
v(a)= \begin{cases}v_{0}(a) & a \leq a_{0} \\ v_{0}\left(a_{0}\right) & a_{0} \leq a \leq a_{0}+L-L_{0} \\ v_{0}\left(a+L_{0}-L\right) & a_{0}+L-L_{0} \leq a \leq L\end{cases}
$$

Then $v_{a}$ is a non-negative $L^{1}$ function, vanishing on $\left[a_{0}, a_{0}+L-L_{0}\right]$ and with $\left\|v_{a}\right\|_{1}=\ell$. It is routine to approximate $v_{a}$ by positive $v_{a}^{j}$ in $L^{1}$ and with $\left\|v_{a}^{j}\right\|_{1}=\ell$. Define $v(a)=\int_{0}^{a} v_{a}^{j}$ to get a homeomorphic mapping of finite distortion $g^{j}(w)=$ $v^{j}(a)+i b$. Notice that $g^{j} \rightarrow g$ uniformly in $\mathscr{W}^{1,1}([0, L] \times[0,1])$. Set

$$
f^{j}=\left(g^{j}\right)^{-1}:[0, \ell] \times[0,1] \rightarrow[0, L] \times[0,1]
$$


The mappings $f^{j}$ are surjective homeomorphisms of finite distortion. We calculate, with the change of variables $g^{j}(w)=z$,

$$
\begin{aligned}
\int_{\mathbf{Q}_{1}} \varphi\left(\mathbb{K}\left(z, f^{j}\right)\right) \lambda(z) d z & =\int_{\mathbf{Q}_{2}} \varphi\left(\frac{\left\|D f^{j}\left(g^{j}\right)\right\|^{2}}{J\left(g^{j}, f^{j}\right)}\right) \lambda\left(g^{j}(w)\right) J\left(w, g^{j}\right) d w \\
& =\int_{\mathbf{Q}_{2}} \varphi\left(\left\|\left(D g^{j}(w)\right)^{-1}\right\|^{2} J\left(w, g_{j}\right)\right) \lambda\left(g^{j}(w)\right) J\left(w, g^{j}\right) d w \\
& =\int_{\mathbf{Q}_{2}} \varphi\left(v_{a}^{j}(a)+\frac{1}{v_{a}^{j}(a)}\right) \lambda\left(v^{j}(a)\right) v_{a}^{j}(a) d a \\
& \rightarrow \int_{\mathbf{Q}_{2}} \varphi\left(v_{a}(a)+\frac{1}{v_{a}(a)}\right) \lambda(v(a)) v_{a}(a) d a \\
& =\int_{\left[0, L_{0}\right] \times[0,1]} \varphi\left(\left(v_{0}\right)_{a}(a)+\frac{1}{\left(v_{0}\right)_{a}(a)}\right) \lambda\left(\left(v_{0}\right)(a)\right)\left(v_{0}\right)_{a}(a) d a \\
& =\int_{[0, \ell] \times[0,1]} \varphi\left(\left(u_{0}\right)_{x}(x)+\frac{1}{\left(u_{0}\right)_{x}(x)}\right) \lambda(x) d x \\
& =\int_{\mathbf{Q}_{1}} \varphi\left(\mathbb{K}\left(z, f_{0}\right)\right) \lambda(z) d z
\end{aligned}
$$

\section{The Nitsche Phenomenon}

Before moving on to discuss the theory in more generality we provide a couple of interesting applications based around the classical Nitsche problem.

Theorem 7.10 strongly motivates us to study the ordinary differential equation (7.33) for solutions will identify minima of out Nitsche and Grötzsch type problems. Note also that the transformation from the Nitsche type problem to the Grötzsch problem yields a significantly simpler equation to study - in fact it is not really an ODE at all.

8.1. Weighted mean distortion. Let us first observe how the Nitsche phenomenon arises, here we have (ignoring multiplicative constants) $\lambda(x)=e^{4 \pi x}$ as $\eta(w)=1$. We have

So

$$
\begin{gathered}
1-\frac{1}{u_{x}^{2}(x)}=\alpha e^{-4 \pi x}, \quad u_{x}(x)=\frac{1}{\sqrt{1-\alpha e^{-4 \pi x}}} \\
u(x)=\int \frac{e^{2 \pi x} d x}{\sqrt{e^{4 \pi x}-\alpha}}=\frac{1}{2 \pi} \int \frac{d t}{\sqrt{t^{2}-\alpha}}, \quad t=e^{2 \pi x}
\end{gathered}
$$

$$
u(x)=\frac{1}{2 \pi} \log \left(\frac{e^{2 \pi x}+\sqrt{e^{4 \pi x}-\alpha}}{1+\sqrt{1-\alpha}}\right), \quad \alpha \neq 0
$$

noting $u(0)=0$. Recall $u:[0, \ell] \rightarrow[0, L]$ and we must solve $u(\ell)=L$, that is

$$
L=\frac{1}{2 \pi} \log \left(\frac{e^{2 \pi \ell}+\sqrt{e^{4 \pi \ell}-\alpha}}{1+\sqrt{1-\alpha}}\right)
$$

by choice of our free parameter $\alpha$. Notice that $\alpha$ is not bounded from below, and as $\alpha \rightarrow-\infty$ we can make the right hand side of (8.1) as small as we like. Thus 
there is always a minimiser if $L \leqslant \ell$. If $\alpha>0$ we see that (7.33) requires $\alpha<1$ so that

$$
L<\frac{1}{2 \pi} \log \left(\frac{e^{2 \pi \ell}+\sqrt{e^{4 \pi \ell}-1}}{1+\sqrt{1-\alpha}}\right)
$$

and when unwound, these are precisely the Nitsche bounds.

For more general weights $\lambda(x)$,

$$
1-\frac{1}{u_{x}^{2}(x)}=\frac{\alpha}{\lambda(x)}, \quad u_{x}(x)=\sqrt{\frac{\lambda(x)}{\lambda(x)-\alpha}}
$$

and we must typically study the behaviour of an integral like

$$
u(x)=\int_{0}^{\ell} \sqrt{\frac{\lambda(x)}{\lambda(x)-\alpha}} d x .
$$

Again, as $\alpha \rightarrow-\infty$ and if $\lambda$ is not too bad, we can make this integral as small as we wish. Notice that $\alpha / \lambda(x)<1$, so if we put $\lambda_{0}=\min _{x \in[0, \ell]} \lambda(x)$, then this integral is dominated by the one with the choice $\alpha=\lambda_{0}$ and the issue is to decide whether

$$
\int_{0}^{\ell} \sqrt{\frac{\lambda(x)}{\lambda(x)-\lambda_{0}}} d x<\infty .
$$

If this integral is finite, then we will observe Nitsche type phenomenon; nonexistence of minima outside a range of moduli.

Supposing that $\lambda_{0}>0$, the principal issue concerns the integral

$$
\int_{0}^{\ell} \frac{d x}{\sqrt{\lambda(x)-\lambda_{0}}}<\infty
$$

and without going into excessively fine details, convergence will require that

$$
\lambda(t) \approx \lambda_{0}+t^{2 s}, \quad s<1
$$

near the minimum.

In particular, if $\lambda$ is a smooth positive weight and $\lambda^{\prime}(x)=0$ at it's minimum (which may well occur at the endpoints in which case we choose the appropriate left or right derivative), then we can always solve the deformation problem.

8.2. $\varphi^{\prime}$ unbounded; $p>1$. We show that if the convex function $\varphi$ has unbounded derivative, then there is always a minimiser, with mild assumptions on the weight function $\lambda$. In particular we do not see the Nitsche phenomenon for the $L^{p}$-norms of mean distortion. First observe that when $\varphi$ is smooth and convex increasing, the function

$$
F(t)=\left(1-\frac{1}{t^{2}}\right) \varphi^{\prime}\left(t+\frac{1}{t}\right)
$$

is increasing for $t>0$, indeed

$$
F^{\prime}(t)=\frac{2}{t^{3}} \varphi^{\prime}\left(t+\frac{1}{t}\right)+\left(1-\frac{1}{t^{2}}\right)^{2} \varphi^{\prime \prime}\left(t+\frac{1}{t}\right)>0
$$

Next, if $\varphi^{\prime}$ is unbounded, it is monotone and then

$$
\lim _{t \searrow 0} F(t)=-\infty, \quad \lim _{t \rightarrow+\infty} F(t)=+\infty
$$


The intermediate value theorem implies that for each $x \in(0, \ell)$ and $\alpha \in \mathbb{R}$ we can find $t_{x}>0$ so that $F\left(t_{x}\right)=\alpha / \lambda(x)$. We then define a function $v_{\alpha}$ by the rule $v_{\alpha}(x)=t_{x}>0$. Then $v$ is a positive function which certainly satisfies

$$
\lambda(x)\left(1-\frac{1}{v^{2}(x)}\right) \varphi^{\prime}\left(v(x)+\frac{1}{v(x)}\right)=\alpha
$$

The regularity of the function $v_{\alpha}$ depends on that of $\lambda$. The function $u$ that we are looking for defines the mapping $f$ is an antiderivative of $v$. For $f$ to be a mapping of finite distortion, we'll need that $u$ is absolutely continuous. These conditions are all easily seen to be true if $\lambda$ (and hence $v_{\alpha}$ ) is piecewise continuous.

We then define

$$
u_{x}(x)=v_{\alpha}(x)
$$

If $\lambda$ is bounded and bounded away from 0 , then it is easy to see that $v_{\alpha}$ is uniformly large when $\alpha$ is chosen large, while $v_{\alpha}$ is uniformly small if $\alpha$ is chosen large and negative. Further

$$
u(x)=\int_{0}^{x} v_{\alpha}(s) d s
$$

depends continuously on $\alpha$ (as $v_{\alpha}$ depends piecewise continuously). Thus $u(\ell)$ can be made to assume any positive value - in particular we can solve $u(\ell)=L$, and so we don't see the Nitsche phenomenon. Here is a theorem summarising this discussion. The reader will see that we have not striven for maximum generality.

Theorem 8.1. Let $\lambda(x)$ be a piecewise continuous positive weight bounded and bounded away from 0 . Let $\varphi:[1, \infty) \rightarrow[0, \infty)$ be smooth and convex increasing with $\varphi^{\prime}(s)$ unbounded as $s \rightarrow \infty$. Then the minimisation problem

$$
\min _{f \in \mathcal{F}} \int_{\mathbf{Q}_{1}} \varphi(\mathbb{K}(z, f)) \lambda(x)|d z|^{2}
$$

has a unique solution of the form $f(z)=u(x)+i y$. Here $\mathcal{F}$ is the family of all mappings of finite distortion satisfying the boundary conditions described in 7.2

We then have the following corollary about the weighted $L^{p}$-norms of distortion functions.

Corollary 8.2. Let $\lambda(x)$ be a piecewise continuous positive weight bounded and bounded away from 0 . Then the minimisation problem

$$
\min _{f \in \mathcal{F}} \int_{\mathbf{Q}_{1}} \mathbb{K}^{p}(z, f) \lambda(x)|d z|^{2}
$$

has a unique solution of the form $f(z)=u(x)+i y$. Here $\mathcal{F}$ is the family of all mappings of finite distortion satisfying the boundary conditions described above.

8.3. Critical case: $\varphi^{\prime}$ bounded. Examining the above argument we see that in this case we can always find a solution to the minimisation problem of the given form if $L<\ell$ by varying $\alpha$ among negative values, $\alpha=0$ produces the identity mapping. However, there are further subtleties. The reader will quickly get to a condition on the integrability of $\psi\left(\lambda_{0} / \lambda(x)\right)$ where $\psi$ is the inverse of the bounded increasing function $t \mapsto \varphi^{\prime}\left(t+t^{-1}\right)\left(1-t^{-2}\right)$ with $\lambda_{0}=\min _{[0, \ell]} \lambda$. Let us give two 
illustrative examples in the standard (Nitsche) case with $\ell=1, \lambda(x)=e^{-4 \pi x}$. We may assume that $\varphi^{\prime}(t) \nearrow 1$ and the limiting case $\alpha=e^{4 \pi}$ :

Case: $\varphi(t)=t-\log (t), \varphi^{\prime}(t)=1-\frac{1}{t}, a=a(x)=e^{4 \pi(x-1)} \leqslant 1$.

We choose $u_{x}$ to be the largest real root of the polynomial:

$$
\begin{aligned}
\left(1-\frac{1}{t+t^{-1}}\right)\left(1-\frac{1}{t^{2}}\right) & =a \\
p(t)=-1+t-a t^{2}-t^{3}+(1-a) t^{4} & =0 .
\end{aligned}
$$

Since

$$
p\left(\frac{1}{1-a}\right)=-1+\frac{1}{1-a}-\frac{a}{(1-a)^{2}}-\frac{1}{(1-a)^{3}}+\frac{1}{(1-a)^{3}}=-\frac{a^{2}}{(1-a)^{2}}<0
$$

the largest real root $u_{x}(x)>1 /(1-a(x))$ and

$$
\int_{0}^{x} u_{y}(y)>\int_{0}^{x} \frac{1}{1-e^{4 \pi(y-1)}} \approx \frac{1}{4 \pi} \log \left(\frac{1}{1-x}\right)
$$

and this diverges as $x \rightarrow 1$. Therefore with appropriate choice of $\alpha$ we can always solve $u(0)=0$ and $u(1)=L$. Hence there is no Nitsche phenomenon.

Case: $\varphi(t)=t+\frac{1}{(p-1) t^{p-1}}, p>0, p \neq 1$.

We have $\varphi^{\prime}(t)=1-\frac{1}{t^{p}}, 0<a=a(x)=e^{-4 \pi x}<1$ for $0<x<1$, and hence $u_{x}$ is the largest real root of the polynomial

$$
P(t)=\left(1-\frac{1}{\left(t+t^{-1}\right)^{p}}\right)\left(1-\frac{1}{t^{2}}\right)-a=0 .
$$

Note that when $t>0, P(t)$ is a continuous monotonically increasing function of $t$. Also note that $P(1)=-a<0$, and $\lim _{t \rightarrow \infty} P(t)=1-a>0$, so that $P$ has exactly one real positive root $u_{x}>1$.

First let us deal with $0<p<1$. Observe that

$$
\left(1-(1-a)^{2}\right)\left(\left(1+(1-a)^{2}\right)-(1-a)\right)-a\left(1+(1-a)^{2}\right)=-a^{2}(1-a)^{2}<0 .
$$

This may be rewritten as

$$
\left(1-\left(\frac{1}{1-a}\right)^{-2}\right)\left(1-\frac{1}{\frac{1}{1-a}+\frac{1-a}{1}}\right)-a<0
$$

Now using the fact that $0<p<1$, we see that

$$
P\left(\frac{1}{1-a}\right)=\left(1-\frac{1}{\left(\frac{1}{1-a}\right)^{2}}\right)\left(1-\frac{1}{\left(\frac{1}{1-a}+\frac{1-a}{1}\right)^{p}}\right)-a<0
$$

and hence the largest real root $u_{x}>1 /(1-a)$. The integral of the right hand side diverges (see the reasoning for the case $\varphi^{\prime}=1-t^{-1}$ ). Thus with appropriate choice for $\alpha$ we can always solve $u(0)=0, u(1)=L$ and therefore we see no Nitsche phenomenon for $p<1$.

Next, take $p \geqslant 2$. Recall (8.7). Note that $\left(t+\frac{1}{t}\right)^{p}>\left(t+\frac{1}{t}\right)^{2}>t^{2}$. Set $Q(t)$ as

$$
P(t)=\left(1-\frac{1}{\left(t+t^{-1}\right)^{p}}\right)\left(1-\frac{1}{t^{2}}\right)-a>\left(1-\frac{1}{t^{2}}\right)^{2}-a=Q(t), \quad t>1 .
$$


The largest real root of $P(t)$ is therefore dominated by the largest real root of $Q(t)$. Solving $Q(t)=0$ gives

$$
\int_{0}^{1} u_{x} d x<\int_{0}^{1} \frac{1}{\sqrt{1-e^{-2 \pi x}}} d x=\log \left(e^{\pi}+\sqrt{e^{2 \pi}-1}\right),
$$

a finite number. Therefore, when $p \geqslant 2, u_{x}(x)$ is dominated by an integrable function and we must see the Nitsche phenomenon. It is no coincidence that the value of the integral here is strongly reminiscent of that for the Nitsche case (8.1); the integrands for that case and the estimate here are very similar.

It remains to cover the case where $1<p<2$. Note that for $p>1,1-\frac{1}{\left(t+t^{-1}\right)^{p}}>$ $1-\frac{1}{t^{p}}$, and for $p<2,1-\frac{1}{t^{2}}>1-\frac{1}{t^{p}}$. Therefore the polynomial

$$
P(t)=\left(1-\frac{1}{\left(t+t^{-1}\right)^{p}}\right)\left(1-\frac{1}{t^{2}}\right)-a>\left(1-\frac{1}{t^{p}}\right)^{2}-a=Q(t)
$$

and the largest real root of $P(t)$ is again dominated by the largest real root of $Q(t)$. Solving $Q(t)=0$ yields $u_{x}<(1-\sqrt{a(x)})^{-1 / p}$. Near $x=0, \sqrt{a(x)}=e^{-2 \pi x} \approx$ $1-2 \pi x$ and so

$$
\int_{0}^{1} \frac{1}{(1-\sqrt{a(x)})^{1 / p}} d x \approx\left(\frac{1}{2 \pi}\right)^{1 / p} \int_{0}^{1} \frac{1}{x^{1 / p}} d x
$$

which converges if and only if $p>1$. Therefore in this case, too, $u_{x}$ is dominated by an integrable function and we must see a critical Nitsche-type phenomenon.

\section{Teichmüller's Problem.}

In the preceding (frictionless) examples we have seen the case of minimisers of $L^{p_{-}}$ mean distortion that $p=1$ is rather special and that $p \in(1, \infty]$ are similar in that extremal mappings exist and are regular. We just mention here a related problem with boundary values in which the exact opposite occurs. Namely $p \in[1, \infty)$ have the same nature (nonexistence of minimisers, see $[\mathbf{2 8}]$ for the case $p=1$.) while $p=\infty$ has minimisers. Teichmüller's problem for mean distortion is to identify for $r>0$,

$$
\inf \|\mathbb{K}(z, f)\|_{L^{p}(\mathbb{D})}
$$

and show a minimiser exists. Here the infimum is taken over all mappings $f$ : $\mathbb{D} \stackrel{\text { onto }}{\longrightarrow} \mathbb{D}$ of finite distortion with $f \in \mathscr{W}_{\text {loc }}^{1,2}(\mathbb{D}), f(0)=r$, and that can be extended to a homeomorphism of the closed disk onto itself with $f \mid \partial \mathbb{D}=I d$. The classical Teichmüller problem is $p=\infty$ where the maximal distortion is employed instead of the mean distortion and minimisers exist and are of Teichmüller type. That is $\mu=k \bar{\phi} / \phi$ where $\phi$ is meromorphic with a pole of order 1 at $r$. However, for $1 \leq p<\infty$ minimisers exist in a weak sense, and there is an associated AhlforsHopf meromorphic quadratic differential with a pole of order 1 , but these minimisers can never be locally quasiconformal except in the trivial case $r=0$ and $f(z)=z$, [31]. 


\section{References}

[1] K. Astala, T. Iwaniec, and G. Martin, Elliptic partial differential equations and quasiconformal mappings in the plane, Princeton University Press, 2009.

[2] K. Astala, T. Iwaniec, and G. Martin, Deformations of annuli with smallest mean distortion, Arch. Ration. Mech. Anal. 195 (2010), no. 3, 899-921.

[3] K. Astala, T. Iwaniec, G. J. Martin, and J. Onninen, J. Extremal mappings of finite distortion. Proc. London Math. Soc. (3) 91 (2005), no. 3, 655-702.

[4] J. M. Ball, Convexity conditions and existence theorems in nonlinear elasticity, Arch. Rational Mech. Anal. 63 (1976/77), no. 4, 337-403.

[5] J. M. Ball, Global invertibility of Sobolev functions and the interpenetration of matter, Proc. Roy. Soc. Edinburgh Sect. A, 88 (1981), 315-328.

[6] J. M. Ball, Discontinuous equilibrium solutions and cavitation in nonlinear elasticity, Philos. Trans. R. Soc. Lond. A 306 (1982) 557-611.

[7] J. M. Ball, J. C. Currie, and P. J. Olver, Null Lagrangians, weak continuity, and variational problems of arbitrary order, J. Funct. Anal. 41 (1981), no. 2, $135-174$.

[8] R. Caccioppoli, Funzioni pseudo-analitiche e rappresentazioni pseudo-conformi delle superfice riemanniane, Ricerche Mat. 2 (1953) 104-127.

[9] G. Choquet, Sur un type de transformation analytique généralisant la représentation conforme et définie au moyen de fonctions harmoniques, Bull. Sci. Math., 69, (1945), 156-165.

[10] M. de Franchis, La piti generale funzione d'invarianza per criteri sufficienti di minim0 con condizioni di Dirichlet per integrali pluridimensionali del primo ordine dipendenti da un vettore a piu componenti, Atti Accad. Naz. Lincei Rend. Cl. Sci. Fis. Mat. Natur., 37 (8) (1964), 129-140.

[11] D. G. B. Edelen, The null set of the Euler-Lagrange operator, Arch. Rational Mech. Anal. 11 (1962) 117-121.

[12] J. Eells and L. Lemaire, A Report on Harmonic Maps, Bulletin London Math. Soc., 10, (1978), 1-68.

[13] J. Eells and L. Lemaire, Another report on harmonic maps, Bulletin London Math. Soc., 20, (1988), 385-524.

[14] J. Eells and J.Y. Sampson, Harmonic Mappings of Riemannian Manifolds, American Journal of Mathematics, 86, (1964), 109-160.

[15] J.L. Ericksen, Nilpotent energies in liquid crystal theory, Arch. Rat. Mech. Anal., 10, (1962), 189-96

[16] L. Greco, T. Iwaniec, and U. Subramanian, Another approach to biting convergence of Jacobians, Illinois Journal of Mathematics, vol. 47, No. 5 (2003), pp. $815-830$.

[17] S. Hencl, and P. Koskela, Lectures on mappings of finite distortion, Lecture Notes in Mathematics, 2096, Springer, Cham, 2014. xii+176 pp

[18] F. Hang and F. Lin, Topology of Sobolev mappings II, Acta Math. 191 (2003), $55-107$.

[19] T. Iwaniec, A. Lutoborski, Integral Estimates for Null Lagrangians, Arch. Rational Mech. Anal. 125 (1993), 25-79.

[20] T. Iwaniec, L. V. Kovalev, and J. Onninen, The Nitsche conjecture, J. Amer. Math. Soc. 24 (2011), no. 2, 345-373. 
[21] T. Iwaniec and G. Martin, Geometric Function Theory and Non-linear Analysis, Oxford Mathematical Monographs, Oxford University Press, (2001).

[22] T. Iwaniec, G.J. Martin and J. Onninen On Minimisers of $L^{p}$-mean Distortion, Computational Methods and Function Theory, 14, (2014), 399-416.

[23] T. Iwaniec and J. Onninen, $\mathcal{H}^{1}$-estimates of Jacobians by subdeterminants, Mathematische Annalen 324, (2002), 341-358.

[24] T. Iwaniec and J. Onninen, $n$-Harmonic mappings between annuli, Mem. Amer. Math. Soc. 218 (2012).

[25] T. Iwaniec and J. Onninen, Mappings of smallest mean distortion and freeLagrangians, Ann. Sc. Norm. Super. Pisa Cl. Sci., 20, (2020), 1-106.

[26] H. Kneser, Lösung der Aufgabe 41, Jahresber. Deutsch. Math.-Verein., 35, (1926), 123-124.

[27] J.W. Landers, Invariant multiple integrals in the Calculus of Variations Contributions to the Calculus of Variations, (1938-1941) (Chicago: University of Chicago Press) pp 175-208.

[28] G.J. Martin, The Teichmmüller problem for mean distortion, Ann. Acad. Sci. Fenn. Math, 34, (2009) , 233-247.

[29] G.J. Martin, Harmonic degree 1 maps are diffeomorphisms: Lewy's theorem for curved metrics, Transactions of the American Math. Soc., 368, 647-658.

[30] G.J. Martin and M. McKubre-Jordens, Deformations with smallest weighted $L^{p}$ average distortion and Nitsche-type phenomenon, J. Lond. Math. Soc., 85, (2012), 282-300.

[31] G.J. Martin and C. Yao The Teichmüller problem for $L^{p}$-means of distortion, Math. arXiv:2107.07660 (2021) to appear.

[32] C. B. Morrey, Quasi-convexity and the lower semicontinuity of multiple integrals, Pacific J. Math. 2, (1952). 25-53.

[33] S. Müller, S. Qi, T. and Yan, B.S., On a new class of elastic deformations not allowing for cavitation, Ann. Inst. H. Poincaré Anal.. Non Lineaire 11 (2) (1994), 217-97243.

[34] J. Nash, $C^{1}$ isometric imbeddings, Ann. Math. (2) 60, (1954) 383-396.

[35] J.C.C. Nitsche, On the modulus of doubly connected regions under harmonic mappings, Amer. Math. Monthly, 69, (1962), 781-782.

[36] T. Radó, Aufgabe 41., Jahresber. Deutsch. Math.-Verein., 35, (1926), 49.

[37] H. Rund, TheHamilton-Jacobi theory in the Calculus of Variations, London:vanNostrand, 1966.

[38] K. Zhang, Biting theorems for the Jacobians and their applications, Ann. Inst. Henri Poincaré 7 no. 4, (1990), 345-365. 
TI: Department of Mathematics

Syracuse University

Syracuse

NY, USA

tiwaniec@syr.edu

GM: NZ Institute for Advanced Study

Massey University

Auckland

New Zealand

G.J.Martin@massey.ac.nz

JO: Department of Mathematics

Syracuse University

Syracuse

NY, USA

jkonnine@syr.edu 